\title{
On the stability of stationary states in general road networks
}

\author{
Wen-Long Jin *
}

December 17, 2016

\begin{abstract}
In (Jin, 2015), with a discrete map in critical demand levels, it was proved that there exist stationary states for the kinematic wave model of general road networks with constant origin demands, route choice proportions, and destination supplies. In this study we further examine the stability property of stationary states with the same map, and the results will help us to understand the long-term trend of a network traffic system. We first review a network kinematic wave model and properties of stationary states on a link, define the criticality of junctions in stationary states, and discuss information propagation in stationary states on links and junctions. We then present the map and examine information propagation in the map. We apply the map to analytically study the stability of stationary states on ring roads and divergemerge networks with circular information propagation and compare them with results obtained from the Poincaré map (Jin, 2013). We further study the stability property of general stationary states in a grid network. We find that the stability of fixed points of the map is the same as that of stationary states in a network, and the new approach is more general than the Poincaré map approach. We conclude the study with future directions and implications.
\end{abstract}

Key words: Network kinematic wave model; stationary states; map in critical demand levels; criticality of junctions; stability.

\section{Introduction}

One of the core tasks in road transportation research is to analyze, control, manage, plan, and design road networks during peak periods. Since "the traffic demand and origin-destination

*Department of Civil and Environmental Engineering, California Institute for Telecommunications and Information Technology, Institute of Transportation Studies, 4000 Anteater Instruction and Research Bldg, University of California, Irvine, CA 92697-3600. Tel: 949-824-1672. Fax: 949-824-8385. Email: wjin@uci.edu. Corresponding author 
desires are relatively constant over the time period" (Wattleworth, 1967), it has been assumed that traffic patterns are relatively stationary during a peak period in many transportation network problems. Such an assumption has been underlying the static traffic assignment problem (Beckmann et al., 1956; Merchant and Nemhauser, 1978), the local and global control problem of a freeway system (Wattleworth, 1967), the existence of a networkwide macroscopic fundamental diagram (MFD) (Godfrey, 1969; Jin et al., 2013), the network flow problem (Potts and Oliver, 1972), and the integrated traffic assignment and ramp metering problem (Payne and Thompson, 1974; Yang and Yagar, 1995; Yang and Lam, 1996). Therefore it is important to understand properties of such stationary states.

In the literature, there are very few studies on the definition, existence, stability, and other properties of stationary states in road networks. In (Daganzo, 1996, 2007), the existence and stability properties of gridlock states were discussed for both beltway and arterial networks with kinematic wave models. Such gridlock states are a special type of stationary states, since a gridlocked network will always be in the gridlock state if no mitigation strategies are implemented. In (Jin, 2012C), stationary states were first defined and then solved by enumeration for a diverge-merge network within the framework of a network kinematic wave model. In (Jin, 2013), a Poincaré map in fluxes was derived for stationary states in beltway and diverge-merge networks with circular information (wave) propagation and applied to analyze their stability property. In (Jin, 2015), some observations on the Los Angeles freeway network during the morning peak hours on June 18, 2013 were presented to support that the locations and sizes of queues are nearly time-independent; i.e., it is a reasonable assumption that traffic is stationary during peak periods. Further, the existence of stationary states was proved for a network kinematic wave model with constant origin demands, route choice proportions, and destination supplies; in particular, a discrete map in critical demand levels was constructed for general road networks, and the existence of its fixed point and, therefore, the corresponding stationary state was proved with Brouwer's fixed point theorem. As in other dynamical systems, the stability property of stationary states is also fundamentally important, as it determines the long-term trend of a network traffic system. However, there exists no approach to analyze the stability property of stationary states in general road networks.

In this study, we attempt to fill the gap by investigating the stability property of stationary states in general road networks with the help of the new map in critical demand levels developed in (Jin, 2015). One objective is to show that the stability property of the map's fixed points is the same as that of the corresponding stationary states. Another objective is to show that the new approach based on the map in critical demand levels is more general than the Poincaré map approach in (Jin, 2013) as it applies to road networks of any structure. To achieve these objectives, we first discuss properties of stationary states, by especially defining the criticality of junctions and discussing information propagation in the new map. Then we analytically derive the stability condition of the map's fixed points for stationary states with circular information propagation in beltway and diverge-merge networks and show the consistency in stability for the new approach and the one based on 
the Poincaré map in (Jin, 2013). With the new approach we further discuss the stability property of general stationary states in a $2 \times 2$ grid network, for which the Poincaré map no longer applies, and verify the results with the Cell Transmission Model (Daganzo, 1995).

The rest of the paper is organized as follows. In Section 2, we review the network kinematic wave model and properties of stationary states on links, define the criticality of junctions, and discuss information propagation on stationary links and junctions. In Section 3 , we present the discrete map in critical demand levels and discuss information propagation in the map regarding inter-dependence of critical demand levels. In Section 4, we analytically study the stability of stationary states in beltway and diverge-merge networks with circular information propagation. In Section 5, we study the stability of stationary states in grid networks. Finally in Section 6 we conclude with some future studies.

\section{A network kinematic wave model and stationary states}

A general road network, e.g., a grid network shown in Figure1, has the following components:

- Links. The set of origin links is denoted by $R$ (dash-dotted red lines), the set of destination links by $W$ (dash-dotted green lines), the set of regular links by $A$ (solid black lines), and the set of all links by $A^{\prime}=R \cup W \cup A$. All the links are directed. The origin and destination links can be dummy links with zero lengths.

- Junctions. The set of junctions is denoted by $J$ (cyan disks). For junction $j \in J$, the set of upstream (incoming) links is denoted by $I_{j}$, and the set of downstream (outgoing) links by $O_{j}$.

- Commodities. The set of commodities is denoted by $\Omega$. In this study, vehicles using the same route (blue dashed line) belong to the same commodity; but we can further differentiate commodities according to vehicle classes and other characteristics. The set of commodities using link $a \in A^{\prime}$ is denoted by $\Omega_{a}$. Without loss of generality, we assume that all routes are acyclic.

For example, the network in Figure 1 has four origin links, four destination links, eight regular links, eight junctions, and 16 routes and commodities.

For link $a$, whose length is $L_{a}$, we denote the longitudinal coordinate by $x_{a} \in\left[0, L_{a}\right]$, which increases in the traffic direction. In addition, at $x_{a}$ and time $t$, the total density, speed, and flow-rate are denoted by $k_{a}\left(x_{a}, t\right), v_{a}\left(x_{a}, t\right)$, and $q_{a}\left(x_{a}, t\right)$, respectively; the density, speed, and flow-rate of commodity $\omega \in \Omega_{a}$ are denoted by $k_{a, \omega}\left(x_{a}, t\right), v_{a, \omega}\left(x_{a}, t\right)$, and $q_{a, \omega}\left(x_{a}, t\right)$, respectively; and the density proportion of commodity $\omega$ is denoted by $\xi_{a, \omega}=k_{a, \omega} / k_{a}$. Hereafter we omit $\left(x_{a}, t\right)$ from the variables unless necessary. 


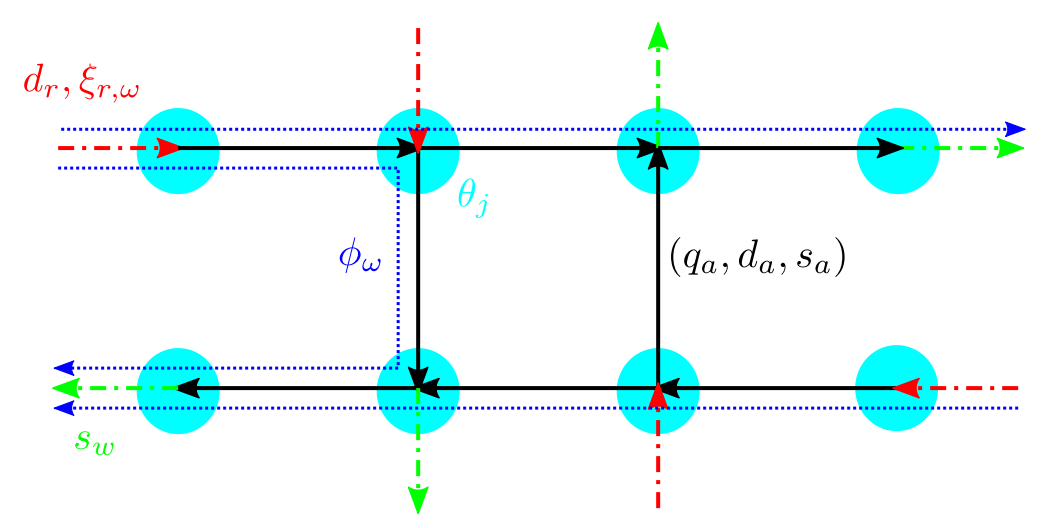

Figure 1: A grid network

\subsection{A network kinematic wave model}

The network kinematic wave theory describes traffic dynamics through the evolution of shock and rarefaction waves on links under given initial and boundary conditions. The boundary conditions include origin demands, $d_{r}(t)(r \in R)$, route choice proportions, $\xi_{r, \omega}(t) \sqrt{1}$, and destination supplies, $s_{w}(t)(w \in W)$.

For link traffic dynamics, the traditional LWR model (Lighthill and Whitham, 1955; Richards, 1956) describes the evolution of traffic density $k_{a}$ with the hyperbolic conservation law:

$$
\frac{\partial k_{a}}{\partial t}+\frac{\partial Q_{a}\left(k_{a}\right)}{\partial x_{a}}=0
$$

Here a speed-density relation in steady states,

$$
v_{a}=V_{a}\left(k_{a}\right)
$$

leads to the fundamental diagram of traffic flow (Greenshields, 1935):

$$
q_{a}=Q_{a}\left(k_{a}\right)=k_{a} V_{a}\left(k_{a}\right) .
$$

Generally, $Q_{a}\left(k_{a}\right)$ is a unimodal function in $k_{a}$ and reaches its capacity, $C_{a}$, when traffic density equals the critical density $k_{a, c}$. If traffic density $k_{a}$ is strictly smaller than, equal to, or strictly greater than the critical density $k_{a, c}$, then we call the traffic state as strictly under-critical (SUC), critical (C), or strictly over-critical (SOC), respectively. An undercritical state (UC) can be SUC or C, and an over-critical state (OC) can be SOC or C. For commodity $\omega$ on link $a$, we have the following multi-commodity LWR model:

$$
\frac{\partial k_{a, \omega}}{\partial t}+\frac{\partial k_{a, \omega} V_{a}\left(k_{a}\right)}{\partial x_{a}}=0
$$

\footnotetext{
${ }^{1}$ Here commodity is the same as route. Thus $\xi_{r, \omega}(t)$ is the proportion of vehicles leaving origin $r$ and choosing route $\omega$ at $t$. Thus it is called the route choice proportion.
} 
Here we assume that vehicles of different commodities follow the first-in-first-out principle (Lebacque, 1996). Therefore, the link-based kinematic wave model (1c) is a system of networked hyperbolic conservation laws, which are neither one-dimensional nor two-dimensional.

A systematic approach to model merging and diverging behaviors macroscopically was proposed in the Cell Transmission Model (CTM) (Daganzo, 1995), based on traffic demand and supply functions (Lebacque, 1996), or sending and receiving flows:

$$
\begin{aligned}
& d_{a}\left(x_{a}, t\right)=D_{a}\left(k_{a}\right) \equiv Q_{a}\left(\min \left\{k_{a}, k_{a, c}\right\}\right), \\
& s_{a}\left(x_{a}, t\right)=S_{a}\left(k_{a}\right) \equiv Q_{a}\left(\max \left\{k_{a}, k_{a, c}\right\}\right),
\end{aligned}
$$

where the traffic demand function, $D_{a}\left(k_{a}\right)$, is non-decreasing in $k_{a}$, and the traffic supply function, $S_{a}\left(k_{a}\right)$, non-increasing in $k_{a}$. We can see that $q_{a}=\min \left\{d_{a}, s_{a}\right\}, C_{a}=\max \left\{d_{a}, s_{a}\right\}$, and $k_{a}$ can be uniquely determined by the congestion level, $d_{a} / s_{a}$, which is a strictly increasing function of $k_{a}$. We denote the function of density in the congestion level by

$$
k_{a}=K_{a}\left(d_{a} / s_{a}\right) .
$$

Therefore, instead of using traffic density $k_{a}$, we can also use traffic demand and supply, $U_{a}=\left(d_{a}, s_{a}\right)$, as state variables. Thus a traffic state is UC if and only if $q_{a}=d_{a} \leq s_{a}=C_{a}$, or equivalently $U_{a}=\left(q_{a}, C_{a}\right)$; a traffic state is $\mathrm{OC}$ if and only if $q_{a}=s_{a} \leq d_{a}=C_{a}$, or equivalently $U_{a}=\left(C_{a}, q_{a}\right)$.

For a general junction $j$ with $m$ upstream links $I_{j}=\{1, \cdots, m\}$ and $n$ downstream links $O_{j}=\{m+1, \cdots, m+n\}$, a macroscopic junction model determines boundary fluxes from upstream demands, turning proportions, and downstream supplies. In (Jin, 2012b), it was shown that such junction flux functions can be used as entropy conditions to pick out unique and physical solutions of the multi-commodity LWR model (1c) at network junctions. In this study, we use the invariant junction model developed in (Jin, 2012a), which substantially simplifies analyses by eliminating interior states from solutions. Here the upstream demands and turning proportions are defined at $\left(L_{a}^{-}, t\right)\left(a \in I_{j}\right)$, downstream supplies at $\left(0^{+}, t\right)$ $\left(b \in O_{j}\right)$, and fluxes at the junction point $\left(L_{a}\right.$ for upstream link $a$ and 0 for downstream link $b)$.

1. Turning proportions: At any time $t$, the turning proportions $\xi_{a \rightarrow b}$ can be calculated from commodity density proportions:

$$
\xi_{a \rightarrow b}=\sum_{\omega \in \Omega_{b}} \xi_{a, \omega}
$$

2. Demand levels:

(a) The demand level of upstream link $a \in I_{j}$ is defined as $\mu_{a}=\frac{d_{a}}{C_{a}}$. 
(b) We define the adjusted average demand level of a non-empty set $A \subseteq I_{j}$ with respect to link $b \in O_{j}$ as

$$
\gamma_{b}\left(A ; s_{b}, \vec{d}, \vec{C}, \vec{\xi}_{b}\right)=\frac{\pi_{b}+\sum_{a \in A} \mu_{a} C_{a \rightarrow b}}{\sum_{a \in A} C_{a \rightarrow b}},
$$

where $C_{a \rightarrow b}=C_{a} \xi_{a \rightarrow b}$, the residual supply $\pi_{b}=s_{b}-\sum_{a \in I_{j}} d_{a} \xi_{a \rightarrow b}=s_{b}-$ $\sum_{a \in I_{j}} \mu_{a} C_{a \rightarrow b}, \vec{d}=\left(d_{a}\right)_{a \in I_{j}}, \vec{C}=\left(C_{a}\right)_{a \in I_{j}}$, and $\vec{\xi}_{b}=\left(\xi_{a \rightarrow b}\right)_{a \in I_{j}}$.

(c) Then the critical demand level for link $b$ is

$$
\Gamma_{b}\left(s_{b}, \vec{d}, \vec{C}, \vec{\xi}_{b}\right)=\max _{A \neq \emptyset, A \subseteq I_{j}} \gamma_{b}\left(A ; s_{b}, \vec{d}, \vec{C}, \vec{\xi}_{b}\right) .
$$

(d) Finally, the critical demand level for junction $j$ is defined as

$$
\theta_{j}=\min _{b \in O_{j}}\left\{1, \Gamma_{b}\right\}
$$

3. Boundary fluxes:

(a) The out-flux of upstream link $a \in I_{j}$ is

$$
g_{a}=\min \left\{d_{a}, \theta_{j} C_{a}\right\}=\min \left\{\mu_{a}, \theta_{j}\right\} C_{a} .
$$

We can see that when link $a$ 's demand level is greater than the junction's critical demand level, then its out-flux is determined by the latter, and the link becomes congested; otherwise, the link is uncongested, and its out-flux equals the demand.

(b) The commodity flux is $\left(a \in I_{j}, b \in O_{j}, \omega \in \Omega_{a} \cap \Omega_{b}\right)$

$$
\phi_{\omega}=q_{b, \omega}=q_{a, \omega}=g_{a} \xi_{a, \omega} .
$$

(c) The in-flux of downstream link $b \in O_{j}$ is

$$
f_{b}=\sum_{a \in I_{j}} g_{a} \xi_{a \rightarrow b}
$$

The above junction model was derived in (Jin, 2012b) from a non-invariant one, in which an upstream link?s outflux is proportional to its demand, and a commodity's flux proportional to its density proportion in the upstream links. Thus it is consistent with the fair merging and FIFO diverging rules. In particular, (4e) leads to the fair merging rule, since an upstream link's out-flux either equals to its demand (when it is uncongested) or is proportional to its capacity (when it is congested). In addition, (9c) leads to the FIFO diverging rule, since all upstream links are in "one-pipe regime" and the commodity fluxes are proportional to their density proportions (Daganzo, 1995). In addition, this model 
captures the queue spillback phenomenon at junctions. For example, in an extreme case when a downstream link $b$ is jammed with $s_{b}=0$ and all turning proportions to this link are positive, then the critical demand level is 0 , and all upstream links become jammed with zero out-fluxes. That is, one jammed downstream link would block all upstream links.

In the junction model (4), the most important quantity is the critical demand level, which separates SOC and UC upstream links. As shown in (Jin, 2012a), an upstream link becomes congested when its demand level is greater than the critical demand level.

\section{$2.2 \quad$ Stationary states on links}

In this study, we consider a road network with time-independent boundary conditions: $d_{r}(t)=d_{r}, \xi_{r, \omega}(t)=\xi_{r, \omega}$, and $s_{w}(t)=s_{w}$. In $($ Jin, 2012c, 2015), it was shown that in stationary states the flow-rate is both spatially and temporally independent on a link; i.e., $q_{a}\left(x_{a}, t\right)=q_{a}$. In particular, the flow-rate equals both the in- and out-fluxes in stationary states. The corresponding density is given by $\left(x_{a} \in\left[0, L_{a}\right]\right) 2$

$$
k_{a}\left(x_{a}, t\right)=H\left(u_{a} L_{a}-x_{a}\right)\left(K_{a}\left(q_{a} / C_{a}\right)-K_{a}\left(C_{a} / q_{a}\right)\right)+K_{a}\left(C_{a} / q_{a}\right),
$$

where $H(\cdot)$ is the Heaviside function, and $u_{a} \in[0,1]$ is the uncongested fraction of the road. Basically the stationary state is a stationary (zero-speed) shock wave and its extreme cases: when $u_{a} \in(0,1)$, it is a zero-speed shock wave connecting an upstream uncongested (UC) state, $K_{a}\left(q_{a} / C_{a}\right)$, to a downstream congested (OC) state, $K_{a}\left(C_{a} / q_{a}\right)$; when $u_{a}=0$, the whole link is congested (OC); when $u_{a}=1$, the whole link is uncongested (UC); when $q_{a}=C_{a}$, the whole link is in a critical (C) state for any $u_{a}$. In this sense, $u_{a}$ defines the criticality of a stationary link, and one can determine the stationary state on a link from its flow-rate and the criticality.

For link $a$, we denote the link supply at the upstream section and the link demand at the downstream section by $s_{a}=s_{a}\left(0^{+}, t\right)$ and $d_{a}=d_{a}\left(L_{a}^{-}, t\right)$, respectively. Thus the stationary state on a link can also be determined by stationary flow-rate, $q_{a}$, link supply, $s_{a}$, and link demand, $d_{a}: q_{a}=s_{a}=d_{a}=C_{a}$ if and only if it's C; $q_{a}=d_{a}<s_{a}=C_{a}$ if and only if it's SUC; $q_{a}=s_{a}<d_{a}=C_{a}$ if and only if it's SOC; $q_{a}<s_{a}=d_{a}=C_{a}$ if and only if it's ZS. Here ZS denotes a zero-speed shock wave connecting an SUC upstream state to an SOC downstream state, which have the same flow-rate $q_{a}$.

In (Jin, 2015), it was shown that a road network can be decoupled into links by introducing an upstream outside demand at $0^{-}, d_{a}^{-}$, and downstream outside supply at $L_{a}^{+}, s_{a}^{+}$, for link $a$. Then one can calculate the flow-rate from

$$
q_{a}=\min \left\{d_{a}^{-}, s_{a}^{+}, C_{a}\right\},
$$

and determine stationary states by following Lemma 2.1. Note that $d_{r}^{-}=d_{r}$ for an origin link $r$ and $s_{w}^{+}=s_{w}$ for a destination link $w$.

\footnotetext{
${ }^{2}$ Refer to Figure 5 of (Jin, 2015) for an illustration of the stationary density distribution on a link.
} 
Lemma 2.1 (Jin, 2015) From the flow-rate, $q_{a}$, the capacity, $C_{a}$, the upstream outside demand, $d_{a}^{-}$, and the downstream outside supply, $s_{a}^{+}$, we can determine the stationary state on a regular link $a \in A$ as follows:

1. If $q_{a}=C_{a}$, the link is stationary at $C$ with $d_{a}=s_{a}=q_{a}=C_{a}$;

2. If $q_{a}=s_{a}^{+}<C_{a}$, and $q_{a}<d_{a}^{-}$, the link is stationary at $S O C$ with $d_{a}=C_{a}>q_{a}=s_{a}$;

3. If $q_{a}<s_{a}^{+} \leq C_{a}$, and the link is stationary at SUC with $d_{a}=q_{a}=d_{a}^{-}<C_{a}=s_{a}$;

4. If $q_{a}<C_{a}, q_{a}=s_{a}^{+}$, and $q_{a}=d_{a}^{-}$, the link is stationary at either SUC, SOC, or ZS.

Note that the fourth scenario is much less likely than the second or third one, since a small disturbance in either $d_{a}^{-}$or $s_{a}^{+}$would drive the stationary state away from it. In addition, when $q_{a}=s_{a}^{+}=d_{a}^{-}$, different stationary states are equivalent to an SOC state. Thus we can consider the fourth scenario as a special case of the second one, and practically we just need to compare $q_{a}$ with $C_{a}$ and $s_{a}^{+}$as in the first three steps. This simplification avoids the calculation of $d_{a}^{-}$.

We can extend Lemma 2.1 to origin and destination links as follows:

- For an origin link $r$, we assume that $d_{r} \leq C_{r}$. Thus from the flow-rate, $q_{r}$, the capacity, $C_{r}$, the origin demand, $d_{r}$, and the downstream outside supply, $s_{r}^{+}$, we can determine its criticality as follows:

1. If $q_{r}=C_{r}$, the origin is stationary at $\mathrm{C}$;

2. If $q_{r}=s_{r}^{+}<d_{r}$, the origin is stationary at SOC;

3. If $q_{r}<s_{r}^{+} \leq C_{r}$, the origin is stationary at SUC;

4. If $q_{r}=s_{r}^{+}=d_{r}<C_{r}$, the origin is stationary at either SUC, SOC, or ZS.

- For a destination link $w$, we assume that $s_{w} \leq C_{w}$. Thus from the flow-rate, $q_{w}$, the capacity $C_{w}$, the upstream outside demand, $d_{w}^{-}$, and the destination supply, $s_{w}$, we can determine its criticality as follows:

1. If $q_{w}=C_{w}$, the destination is stationary at $\mathrm{C}$;

2. If $q_{w}=s_{w}<d_{w}^{-}$, the destination is stationary at SOC;

3. If $q_{w}<s_{w}$, the destination is stationary at SUC;

4. If $q_{w}=s_{w}=d_{w}^{-}<C_{w}$, the destination is stationary at either SUC, SOC, or ZS.

Further from Lemma 2.1 and (6) we have the following corollary regarding information propagation on a stationary link in terms of the propagation of small disturbances in upstream outside demand and downstream outside supply. 
Corollary 2.2 We have the following observations regarding the propagation of small disturbances on a stationary link in upstream outside demand, $d_{a}^{-}$, and downstream outside supply, $s_{a}^{+}$:

1. If a link is stationary at $C$, then small increases in $d_{a}^{-}$and $s_{a}^{+}$will not change the status of the link; but small decreases in either of them will lead to either SUC or SOC states. Thus information can be absorbed, or propagate either forward or backward on $C$ links.

2. If a link is stationary at SUC, then small changes in $s_{a}^{+}$are inconsequential, but the link flow-rate, $q_{a}$, and demand, $d_{a}$, change with $d_{a}^{-}$. Thus information propagates forward on SUC links.

3. If a link is stationary at $S O C$, then small changes in $d_{a}^{-}$are inconsequential, but the link flow-rate, $q_{a}$, and supply, $s_{a}$, change with $s_{a}^{+}$. Thus information propagates backward on SOC links.

4. If a link is stationary at ZS, then small changes in either $d_{a}^{-}$or $s_{a}^{+}$will change the criticality of the link. Thus information can propagate either forward or backward on ZS links.

\subsection{Criticality of stationary junctions}

For junction $j$, we separate $I_{j}$ into two sets: $I_{j}^{+}$for all SOC or ZS links with $q_{a}<d_{a}=C_{a}$, and $I_{j}^{-}$for all SUC or C links with $q_{a}=d_{a}$; we separate $O_{j}$ into two sets: $O_{j}^{+}$for all SOC or $\mathrm{C}$ links with $q_{b}=s_{b}$, and $O_{j}^{-}$for all SUC or ZS links with $q_{b}<s_{b}=C_{b}$. For the purpose of simplicity, we omit $\left(s_{b}, \vec{d}, \vec{C}, \vec{\xi}_{b}\right)$ from the definitions of both $\gamma_{b}(A)$ in (4b) and $\Gamma_{b}$ in (4c).

Lemma 2.3 If $\mathrm{O}_{j}^{+}$is empty, then $\mathrm{I}_{j}^{+}$is. The contrapositive statement is also true: if $\mathrm{I}_{j}^{+}$is not empty, then $O_{j}^{+}$is not.

The proof is given in Appendix A.

From Lemma 2.3, we can define the following three levels of criticality for a stationary junction:

- SUC if all downstream links are SUC or ZS. In this case, $O_{j}^{+}$is empty, and, equivalently, $O_{j}^{-}=O_{j}$. In addition, $I_{j}^{+}$is empty, and all upstream links are $\mathrm{SUC}$ or C; i.e., $I_{j}^{-}=I_{j}$.

- $\mathrm{C}$ if at least one downstream link is SOC or C and no upstream link is SOC or ZS. In this case, $O_{j}^{+}$is not empty, but $I_{j}^{+}$is empty and, equivalently, $I_{j}^{-}=I_{j}$.

- SOC if at least one upstream link is SOC or ZS. In this case, $I_{j}^{+}$is not empty. In addition, $\mathrm{O}_{j}^{+}$is not empty; i.e., at least one downstream link is SOC or C. 
Thus a junction is SUC when both $I_{j}^{+}$and $O_{j}^{+}$are empty; C when $I_{j}^{+}$is empty but $O_{j}^{+}$not; and SOC when both $I_{j}^{+}$and $O_{j}^{+}$are not empty. It is not possible that $O_{j}^{+}$is empty but $I_{j}^{+}$ not, according to Lemma 2.3 .

Next we discuss information propagation at a stationary junction based on two lemmas related to the adjusted average demand level in (4b), the critical demand level in (4c), and the critical demand level in (4d), which were first proved in (Jin, 2012a).

Lemma 2.4 (Jin, 2012a) $\gamma_{b}(A)$ and $\Gamma_{b}$ have the following properties:

1. When $\pi_{b}>0$, then $\gamma_{b}(\{a\})>\mu_{a}$ for any $a \in I_{j}$, and $\Gamma_{b}=\max _{a \in I_{j}} \gamma_{b}(\{a\})>$ $\max _{a \in I_{j}} \mu_{a}$.

2. When $\pi_{b}=0$, then $\gamma_{b}(\{a\})=\mu_{a}$ for any $a \in I_{j}$, and $\Gamma_{b}=\max _{a \in I_{j}} \gamma_{b}(\{a\})=$ $\max _{a \in I_{j}} \mu_{a}$.

3. When $\pi_{b}<0$, then $\gamma_{b}(\{a\})<\mu_{a}$ for any $a \in I_{j}$, and there exists a unique $A_{*} \neq \emptyset$, such that $\Gamma_{b}=\gamma_{b}\left(A_{*}\right), \mu_{a}>\Gamma_{b} \geq \mu_{\alpha}$ for $a \in A_{*}$ and $\alpha \in I_{j} \backslash A_{*}$.

Lemma 2.5 (Jin, 2012a) For $C_{a \rightarrow b}>0, \mu_{a} \in[0,1]$, and $s_{b} \geq 0\left(a \in I_{j}\right.$ and $\left.b \in O_{j}\right)$, $\min _{b \in O_{j}} \Gamma_{b}$ has the following properties:

1. $\min _{b \in O_{j}} \Gamma_{b}>\max _{a \in I_{j}} \mu_{a}$ if and only if $\min _{b \in O_{j}} \pi_{b}>0$.

2. $\min _{b \in O_{j}} \Gamma_{b}=\max _{a \in I_{j}} \mu_{a}$ if and only if $\min _{b \in O_{j}} \pi_{b}=0$.

3. If and only if $\min _{b \in O_{j}} \pi_{b}<0$, there exists a unique non-empty $A_{*} \subseteq I_{j}$ such that

$$
\min _{b \in O_{j}} \Gamma_{b}=\min _{b \in O_{j}} \gamma_{b}\left(A_{*}\right)=\max _{A \neq \emptyset, A \subseteq I_{j}} \min _{b} \gamma_{b}(A),
$$

and

$$
\min _{a \in A_{*}} \mu_{a}>\min _{b \in O_{j}} \Gamma_{b} \geq \max _{a \in I_{j} \backslash A_{*}} \mu_{a} .
$$

Lemma 2.6 If a downstream link $b$ is stationary at $S O C$ or $C$; i.e., if $b \in O_{j}^{+}$or $q_{b}=s_{b}$, then $\theta_{j}=\Gamma_{b}$. Therefore, the critical demand level equals the critical demand level of an $S O C$ or $C$ downstream link. 3

The proof is given in Appendix B.

Theorem 2.7 A junction is stationary at $S U C, C$, or $S O C$, if and only if $\min _{b \in O_{j}} \pi_{b}>,=$ ,$<0$, respectively. Equivalently, a junction is $S U C, C$, or $S O C$, if and only if $\min _{b \in O_{j}} \Gamma_{b}>$ ,$=,<\max _{a \in I_{j}} \frac{d_{a}}{C_{a}}$ respectively.

\footnotetext{
${ }^{3}$ Note that $\theta_{j}=\Gamma_{b}$ may not lead to SOC or $\mathrm{C}$ stationary states on link $b$. A counter example is for a junction with one SUC upstream link and one SUC or ZS downstream link: $q_{b}=d_{a}<s_{b}=C_{b} \leq C_{a}$. In this case, $\pi_{b}>0, \theta_{j}=\Gamma_{b}=\frac{C_{b}}{C_{a}} \leq 1$, and $\frac{d_{a}}{C_{a}}<\Gamma_{b}$.
} 
The proof is given in Appendix C.

From Theorem 2.7, we have the following corollary for information propagation at stationary junctions in terms of the propagation of small disturbances in upstream demands and downstream supplies.

Corollary 2.8 We have the following observations regarding the propagation of information (small disturbances in demands and supplies) at stationary junctions.

1. If a junction is $S U C$, then $q_{a}=d_{a}$ for $a \in I_{j}$ and $q_{b}=\sum_{a \in I_{j}} d_{a} \xi_{a \rightarrow b}$ for $b \in O_{j}$; i.e., all flow-rates are determined by the upstream demands alone. In this case, small disturbances in upstream demands or downstream supplies will not change the criticality of the junction. In addition, small disturbances in upstream demands will propagate to downstream links, but small disturbances in downstream supplies are inconsequential. Thus, information propagates forward at an SUC junction. Further in this case, a small change in the critical demand level will not impact the stationary states on upstream or downstream links, and the junction's critical demand level is equivalent to any number between $\max _{a \in I_{j}} \frac{d_{a}}{C_{a}}$ and 1 and carries no information.

2. A junction initially at $C$ becomes $S U C$ if we decrease any upstream link's demand or increase all SOC/C downstream links' supplies; it becomes SOC if we increase any upstream link's demand or decrease any SOC/C downstream link's supply. Thus, information can propagate forward or backward at a $C$ junction. Further in this case, a mall change in the critical demand level may change the criticality on both upstream and downstream links.

3. If a junction is $S O C$, then for $b \in O_{j}^{+}$

$$
\theta_{j}=\frac{s_{b}-\sum_{\alpha \in I_{j}^{-}} d_{\alpha} \xi_{\alpha \rightarrow b}}{\sum_{a \in I_{j}^{+}} C_{a} \xi_{a \rightarrow b}},
$$

which is determined by the demands of upstream links in $I_{j}^{-}$and the supplies of downstream links in $O_{j}^{+}$. Furthermore, from (4e) we have for $a \in I_{j}^{+}$

$$
q_{a}=C_{a} \theta_{j}
$$

and from (4g) we have for $b \in O_{j}^{-}$

$$
q_{b}=\sum_{a \in I_{j}^{+}} q_{a} \xi_{a \rightarrow b}+\sum_{\alpha \in I_{j}^{-}} d_{\alpha} \xi_{\alpha \rightarrow b} .
$$

Thus information propagates from links in $I_{j}^{-}$and $O_{j}^{+}$to the SOC junction and then to links in $I_{j}^{+}$and $O_{j}^{-}$. In particular, queues on links in $O_{j}^{+}$spill back to $I_{j}^{+}$but starve links in $\mathrm{O}_{j}^{-}$, and higher demands of links in $\mathrm{I}_{j}^{-}$aggravate the situation. Further in this case, a small change in the critical demand level will not change the criticality on all links but change the flow-rates on links in $I_{j}^{+}$. 


\section{A map in critical demand levels and information propagation in the map}

In this section, we first present a map in critical demand levels introduced in (Jin, 2015) and then discuss information propagation in the map around stationary states.

\subsection{A map in critical demand levels}

In (Jin, 2015), a stationary state in a general road network corresponds to a fixed point of the following map in critical demand levels $\left(\vec{\theta}=\left(\theta_{j}\right)_{j \in J}\right.$ and $\theta_{j} \in[0,1]$ for $\left.j \in J\right)$ :

$$
\overrightarrow{\theta^{\prime}}=F(\vec{\theta})
$$

which is defined in the following steps:

1. Outside supplies:

- For link $a \in R \cup A$, the downstream outside supply can be calculated by $\left(C_{r} \geq d_{r}\right.$ for origin link $r$ )

$$
s_{a}^{+}=\theta_{j} C_{a}, \quad a \in I_{j} .
$$

- For destination link $w \in W, s_{w}^{+}=s_{w}$.

2. Link flow-rates:

- The flow-rate of origin $r \in R$ is given by

$$
q_{r}=\min \left\{d_{r}, s_{r}^{+}\right\} .
$$

- Commodity flow-rate is

$$
\phi_{\omega}=\sum_{r \in R} q_{r} \xi_{r, \omega} .
$$

- Link flow-rates on all regular and destination links can be calculated based on the link-route relation $(a \in A \cup W)$

$$
q_{a}=\sum_{\omega \in \Omega_{a}} \phi_{\omega} .
$$

3. The turning proportions at junction $j$ are

$$
\xi_{a \rightarrow b}=\sum_{\omega \in \Omega_{a} \cap \Omega_{b}} \phi_{\omega} / q_{a}
$$

for $a \in I_{j}$ and $b \in O_{j}$. Note that the turning proportions are not well-defined when $q_{a}=0$ in a gridlock state, which has to be treated separately. 
4. We denote the effective link demand and supply by $\delta_{a}$ and $\sigma_{a}$, respectively. For origin $r \in R, \delta_{r}=\min \left\{d_{r}, C_{r}\right\}$; for destination $w \in W, \sigma_{w}=\min \left\{s_{w}, C_{w}\right\}$. For regular link $a \in A$, the effective demand and supply are

$$
\begin{aligned}
\delta_{a} & =\min \left\{q_{a}, C_{a}\right\}, \\
\sigma_{a} & =s_{a}^{+} .
\end{aligned}
$$

5. Based on the effective demands and supplies, the critical demand level at junction $j$ can be updated by:

$$
\theta_{j}^{\prime}=\min _{b \in O_{j}}\left\{1, \Gamma_{b}\left(\sigma_{b}, \vec{\delta}, \vec{C}, \vec{\xi}_{b}\right)\right\}
$$

where $\vec{\delta}=\left(\delta_{a}\right)_{a \in I_{j}}$, and the maximum demand levels $\Gamma_{b}\left(\sigma_{b}, \vec{\delta}, \vec{C}, \vec{\xi}_{b}\right)$ are defined in (4c), except that effective demands and supplies are used in place of actual demands and supplies.

In (Jin, 2015), it was shown that the critical demand level in (9h) is equivalent to that in (4d) in stationary states, and there exists at least one fixed point for (86) with Brouwer's fixed point theorem. Therefore there exists at least one stationary state in a general road network.

In addition, from a fixed point of $\vec{\theta}$, we can calculate stationary states as follows:

1. From (9a), we obtain downstream outside supplies of $a \in R \cup A$ in stationary states, $s_{a}^{+}$.

2. From (9b), (9c), and (9d), we obtain stationary flow-rates on all links $q_{a}\left(a \in A^{\prime}\right)$.

3. From (9e), (9f), and (9g), we obtain stationary turning proportions and effective demands and supplies, from which we can calculate the upstream outside demand of $a \in O_{j}$

$$
d_{a}^{-}=\sum_{\alpha \in I_{j}} \min \left\{d_{\alpha}, \theta_{j \backslash a} C_{\alpha}\right\} \xi_{\alpha \rightarrow a}
$$

where

$$
\theta_{j \backslash a}=\min _{b \in O_{j}, b \neq a}\left\{1, \Gamma_{b}\left(\sigma_{b}, \vec{\delta}, \vec{C}, \vec{\xi}_{b}\right)\right\}
$$

4. For link $a$, given $q_{a}, C_{a}, d_{a}^{-}$, and $s_{a}^{+}$, we can determine the criticality of the link and its link demand and supply from Lemma 2.1. Then the stationary state on the link is obtained. 
Note that in (10) the calculation of $d_{a}^{-}$depends on its upstream links' demands. Thus in a network with a ring road, e.g., a beltway network, such calculations could be circularly dependent. However, as argued following Lemma 2.1, it is unlikely that $s_{a}^{+}=d_{a}^{-}$, and even if $s_{a}^{+}=d_{a}^{-}<C_{a}$ we can still consider the link to be stationary at SOC. That is, we do not need to calculate $d_{a}^{-}$if we are just interested in finding one stationary state without ZS states. But the calculation of $d_{a}^{-}$is necessary if we are interested in finding all possible stationary states, including ZS states.

\subsection{Definition of stability}

In this study, we will further examine the (local) stability property of the fixed points, $\overrightarrow{\theta^{*}}$, of the discrete map in (88) and the corresponding stationary states, $k_{a, \omega}^{*}\left(x_{a}, t\right)$. We first define the stability as follows (LaSalle, 1976; Wiggins and Heck, 2003).

Definition 3.1 (Stability of stationary states) A stationary state, $k_{a, \omega}^{*}\left(x_{a}, t\right)(a \in A, \omega \in$ $\Omega$ ), is Lyapunov stable if, given $\zeta>0$, there exists $z$ such that for all $t>0$ (Here the $\|\cdot\|$ can be the $L^{2}$ norm.)

$$
\sum_{a \in A, \omega \in \Omega}\left\|k_{a, \omega}\left(x_{a}, t\right)-k_{a, \omega}^{*}\left(x_{a}, t\right)\right\|<\zeta
$$

for all initial states $k_{a, \omega}\left(x_{a}, 0\right)$ such that $\sum_{a \in A, \omega \in \Omega}\left\|k_{a, \omega}\left(x_{a}, 0\right)-k_{a, \omega}^{*}\left(x_{a}, t\right)\right\|<z$. That is, for a Lyapunov stable stationary state, the future traffic states will stay around the stationary state under a small perturbation. Further, if $z$ can be chosen so that the stationary state is stable and

$$
\lim _{t \rightarrow \infty} \sum_{a \in A, \omega \in \Omega}\left\|k_{a, \omega}\left(x_{a}, t\right)-k_{a, \omega}^{*}\left(x_{a}, t\right)\right\|=0
$$

then the stationary state is asymptotically stable. That is, for an asymptotically stable stationary state, the future traffic states will converge to the stationary state under a small perturbation. If a stationary state is not Lyapunov stable, then it is unstable.

Here given initial states $k_{a, \omega}\left(x_{a}, 0\right)$, the future traffic states $k_{a, \omega}\left(x_{a}, t\right)$ are obtained through the network kinematic wave model presented in Section 2.1, under the time-independent boundary conditions: $d_{r}(t)=d_{r}, \xi_{r, \omega}(t)=\xi_{r, \omega}$, and $s_{w}(t)=s_{w}$. Since the network kinematic wave model is a nonlinear dynamical system of an infinite dimension, it is very challenging to directly determine the stability of its equilibrium points (stationary states). Therefore we resort to examining the stability of the fixed points of (으), which is a finite dimensional map and can be re-written as

$$
\vec{\theta}(t+1)=F(\vec{\theta}(t)) .
$$


Definition 3.2 (Stability of fixed points) A fixed point, $\vec{\theta}^{*}$, of the discrete map, (8), is Lyapunov stable if, given $\zeta>0$, there exists $z$ such that for all $t>0$ (Here the $\|\cdot\|$ can be the $l^{2}$ norm.)

$$
\left\|\vec{\theta}(t)-\overrightarrow{\theta^{*}}\right\|<\zeta
$$

for all initial states $\left\|\vec{\theta}(0)-\vec{\theta}^{*}\right\|<z$. That is, for a Lyapunov stable fixed point, the future states will stay around the fixed point under a small perturbation. Further, if $z$ can be chosen so that the fixed point is stable and

$$
\lim _{t \rightarrow \infty}\left\|\vec{\theta}(t)-\vec{\theta}^{*}\right\|=0
$$

then the fixed point is asymptotically stable. That is, for an asymptotically stable fixed point, the future states will converge to the fixed point under a small perturbation. If a fixed point is not Lyapunov stable, then it is unstable.

In the following sections, we will establish the equivalence between the stability of stationary states and that of fixed points for specific types of networks. Here the stability property of stationary states is established through simulations, as theoretical analysis is very challenging.

\subsection{Information propagation in the map around a stationary state}

In this subsection, we investigate information propagation in the map (8) regarding the inter-dependence of demand levels. In particular, we consider the dependence of $\theta_{j}^{\prime}$ and $\theta_{j+1}^{\prime}$ on $\theta_{j}$ and $\theta_{j+1}$ along link $a$ that connects an upstream junction $j$ to a downstream junction $j+1$.

Here we only consider the cases when both junctions are SOC and the connecting link is either SUC or SOC for the following reasons:

- First, from Corollary 2.8 we can see that small changes in the critical demand level of an SUC junction do not impact traffic states on the surrounding links, and small changes in the critical demand level of a $\mathrm{C}$ junction may lead to different types of critical states on both upstream and downstream links. That is, the information propagation direction is undetermined for SUC or C junctions. Thus here we only consider SOC junctions, for which the information propagation directions are fixed subject to small disturbances in critical demand levels, upstream demands, or downstream supplies.

- Second, from Corollary 2.2 we can see that the information propagation direction may change on either C or ZS links. Thus we only consider SOC or SUC links, on which the information propagation direction is fixed subject to small disturbances in boundary conditions. 
In addition, we assume that with small disturbances in critical demand levels, the turning proportions $\xi_{a \rightarrow b}\left(a \in I_{j}\right.$ and $\left.b \in O_{j}\right)$ is relatively constant at junction $j$. Then we have the following theorem regarding information propagation in the map, (8) .

Theorem 3.3 For an SUC or SOC link a connecting two SOC junctions $j$ and $j+1$, if it is stationary at $S U C$, then information propagates forward in the map, (9h), in the sense that $\theta_{j+1}^{\prime}$ depends on and decreases with $\theta_{j}$ :

$$
\theta_{j+1}^{\prime}=-\frac{\sum_{\alpha \in I_{j}^{+}} C_{\alpha} \xi_{\alpha \rightarrow a}}{\sum_{\alpha \in I_{j+1}^{+}} C_{\alpha} \xi_{\alpha \rightarrow b}} \theta_{j}+\frac{s_{b}-\sum_{\alpha \in I_{j}^{-}} d_{\alpha} \xi_{\alpha \rightarrow a}-\sum_{\alpha \in I_{j+1}^{-}, \alpha \neq a} q_{\alpha} \xi_{\alpha \rightarrow b}}{\sum_{\alpha \in I_{j+1}^{+}} C_{\alpha} \xi_{\alpha \rightarrow b}}
$$

where $b \in O_{j+1}^{+}$; if it is stationary at $S O C$, then information propagates backward in the map, (9h), in the sense that $\theta_{j}^{\prime}$ depends on and increases with $\theta_{j+1}$ :

$$
\theta_{j}^{\prime}=\frac{C_{a}}{\sum_{\alpha \in I_{j}^{+}} C_{\alpha} \xi_{\alpha \rightarrow a}} \theta_{j+1}-\frac{\sum_{\alpha \in I_{j}^{-}} d_{\alpha} \xi_{\alpha \rightarrow a}}{\sum_{\alpha \in I_{j}^{+}} C_{\alpha} \xi_{\alpha \rightarrow a}} .
$$

The proof is given in Appendix D.

\section{Stability of stationary states with circular informa- tion propagation}

In (Jin, 2013), it was found that there can exist a circular information propagation route in stationary beltway and diverge-merge networks, and solutions of stationary states and the stability property of such stationary states were analyzed with a Poincaré map in fluxes. In this section, we attempt to find stationary states and analyze their stability property with the new map in critical demand levels, (8), as well as results for information propagation in the map in Theorem 3.3. To have fixed information propagation directions subject to small disturbances, we assume all junctions are SOC and all links are either SUC or SOC, as in Theorem 3.3 .

\subsection{Beltway network}

In this subsection, we consider a beltway network shown in Figure2, which has $n$ origins $(R=$ $\{1,3, \cdots, 2 n-1\}), n$ destinations $(W=\{2,4, \cdots, 2 n\}), 2 n$ regular links $(A=\{2 n+1,2 n+$ $2, \cdots, 4 n\})$, and $2 n$ junctions $(J=\{1,2, \cdots, 2 n\})$. Between $n$ origins and $n$ destinations, there are $n^{2}$ acyclic routes and, therefore, $n^{2}$ commodities. We denote the proportion of vehicles from origin $r$ to destination $w$ by $\xi_{r, w}$, where $r \in R, w \in W, \xi_{r, w} \geq 0$, and $\sum_{w \in W} \xi_{r, w}=1$ for $r \in R$.

Here we consider the stationary state when all origin and regular links are SOC, but all destination links are SUC. In this case, all junctions are SOC in stationary states. Then 


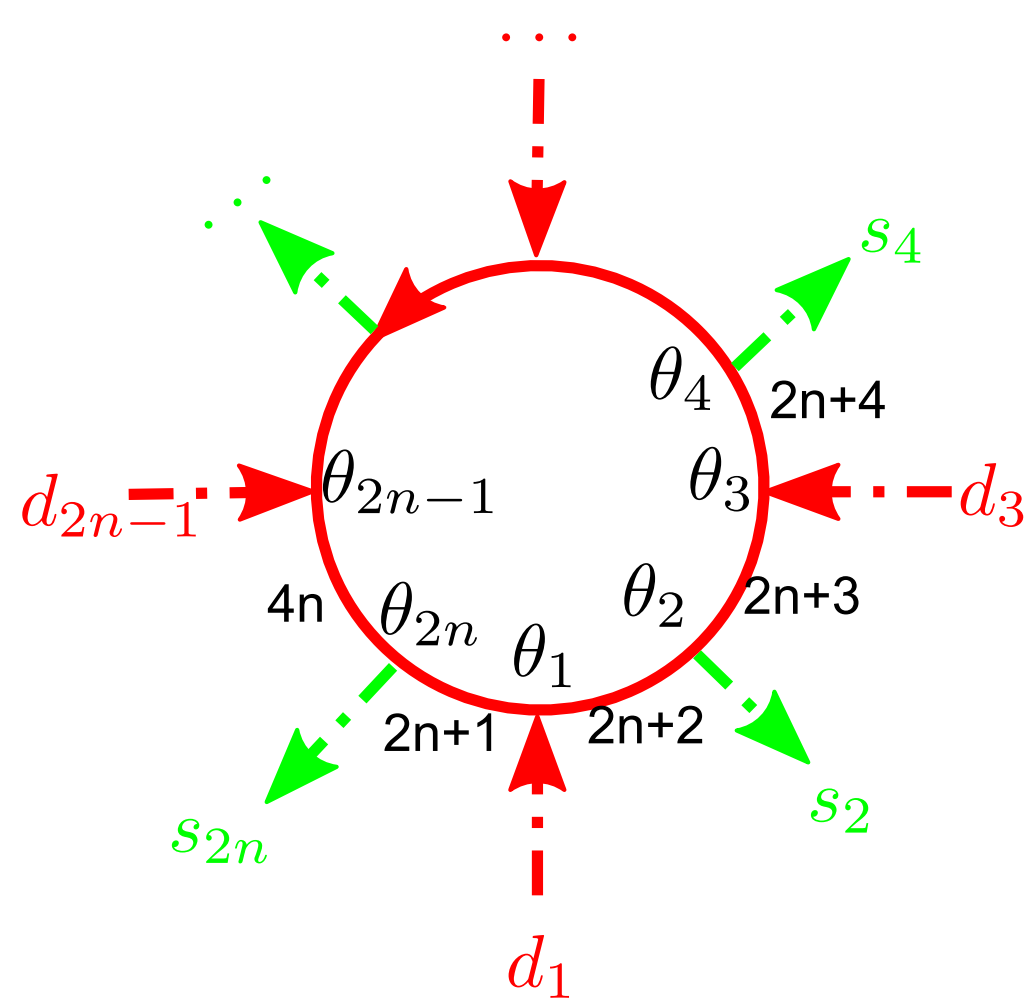

Figure 2: A beltway network: at junction $j$, red links belong to $I_{j}^{+}$or $O_{j}^{+}$, and green links belong to $I_{j}^{-}$or $O_{j}^{-}$

from Theorem 3.3, we can see that information propagates backward on all regular links, and the upstream junction's critical demand level depends on and increases in the downstream junction's, as given in (12). Therefore, (8) can be simplified as the following linear map

$$
\overrightarrow{\theta^{\prime}}=P \vec{\theta}
$$

where $\vec{\theta}=\left[\theta_{1}, \theta_{2}, \cdots, \theta_{2 n-1}, \theta_{2 n}\right]^{T}$, and

$$
P=\left[\begin{array}{lllll}
0 & \frac{C_{2 n+2}}{C_{1}+C_{2 n+1}} & & & \\
& 0 & \frac{C_{2 n+3}}{C_{2 n+2} \xi_{2 n+2 \rightarrow 2 n+3}} & & \\
& & \ddots & \ddots & \\
\vdots & \cdots & 0 & \frac{C_{4 n}}{C_{2 n-1}+C_{4 n-1}} \\
& & \cdots & & 0
\end{array}\right]
$$

We can see that in the map, (13a), all critical demand levels are circularly dependent, corresponding to circular information propagation in the congested beltway network. In 
particular, $\vec{\theta}=0$ is always a fixed point, which corresponds to the gridlock state with jammed regular links.

We observe that $P^{2 n}=\lambda I_{2 n \times 2 n}$, where $I_{2 n \times 2 n}$ is an identity matrix, and

$$
\lambda=\frac{C_{2 n+1}}{C_{1}+C_{2 n+1}} \cdot \frac{1}{\xi_{2 n+2 \rightarrow 2 n+3}} \cdots \cdots \frac{C_{4 n-1}}{C_{2 n-1}+C_{4 n-1}} \cdot \frac{1}{\xi_{4 n \rightarrow 2 n+1}}>0,
$$

where $\frac{C_{2 n+1}}{C_{1}+C_{2 n+1}}$ and $\frac{C_{4 n-1}}{C_{2 n-1}+C_{4 n-1}}$ are the merging ratios at the merging junctions, and $\frac{1}{\xi_{2 n+2 \rightarrow 2 n+3}}$ and $\frac{1}{\xi_{4 n \rightarrow 2 n+1}}$ are the turning proportions at the diverging junctions. Thus, instead of directly analyzing the $2 n$-dimensional map, (13a), with methods given in (Galor, 2007, Chapters 2 and 3 ), we analyze the following equivalent one-dimensional map

$$
\theta_{1}^{(2 n)}=\lambda \theta_{1}
$$

Then from (Galor, 2007, Chapter 1) we have the following observations regarding the new map (15):

1. When $\lambda=1$, there exist an infinite number of fixed points: $\theta_{1}^{*} \in[0,1]$. That is, the beltway network can be stationary at many different SOC states, which may not be gridlocked. All of these stationary states are Lyapunov stable, but not asymptotically stable (LaSalle, 1976).

2. When $\lambda \neq 1$, there exists only one fixed point: $\theta_{1}^{*}=0$, and the beltway network can only be stationary at the gridlock state. In addition, when $\lambda>1$, the gridlock state is unstable; when $\lambda<1$, it is asymptotically stable.

If the beltway network is rotationally symmetric, all merging ratios are equal to $\frac{C_{2 n+1}}{C_{1}+C_{2 n+1}}$, and we denote it by $1-\beta$; and all turning proportions are equal to $\xi_{2 n+2 \rightarrow 2 n+3}$, and we denote it by $1-\xi$. Then $\lambda$ in (14) is equivalent to $\left(\frac{1-\beta}{1-\xi}\right)^{n}$. In this case, the conclusions above are the same as those obtained in (Jin, 2013) through the Poincaré map approach. In this sense, the new map in critical demand levels has the same stability property as the Poincaré map but is more general.

\subsection{Diverge-merge network}

In this subsection, we consider a diverge-merge network shown in Figure 3 , which has $n$ origins $(R=\{1,3, \cdots, 2 n-1\}), n$ destinations $(W=\{2,4, \cdots, 2 n\}), 2 n$ regular links $(A=\{2 n+1,2 n+2, \cdots, 4 n\})$, and $2 n$ junctions $(J=\{1,2, \cdots, 2 n\})$. Between $n$ origins and $n$ destinations, there are $2 n$ acyclic routes and, therefore, $2 n$ commodities. We denote the proportion of vehicles from origin $r$ to its right destination $r+1$ by $\xi_{r}$, where $r \in R$, and $0<\xi_{r}<1$. Then the proportion of vehicles from origin $r$ to its left destination is $1-\xi_{r}$. This network is called a diverge-merge network, because downstream to an origin is 


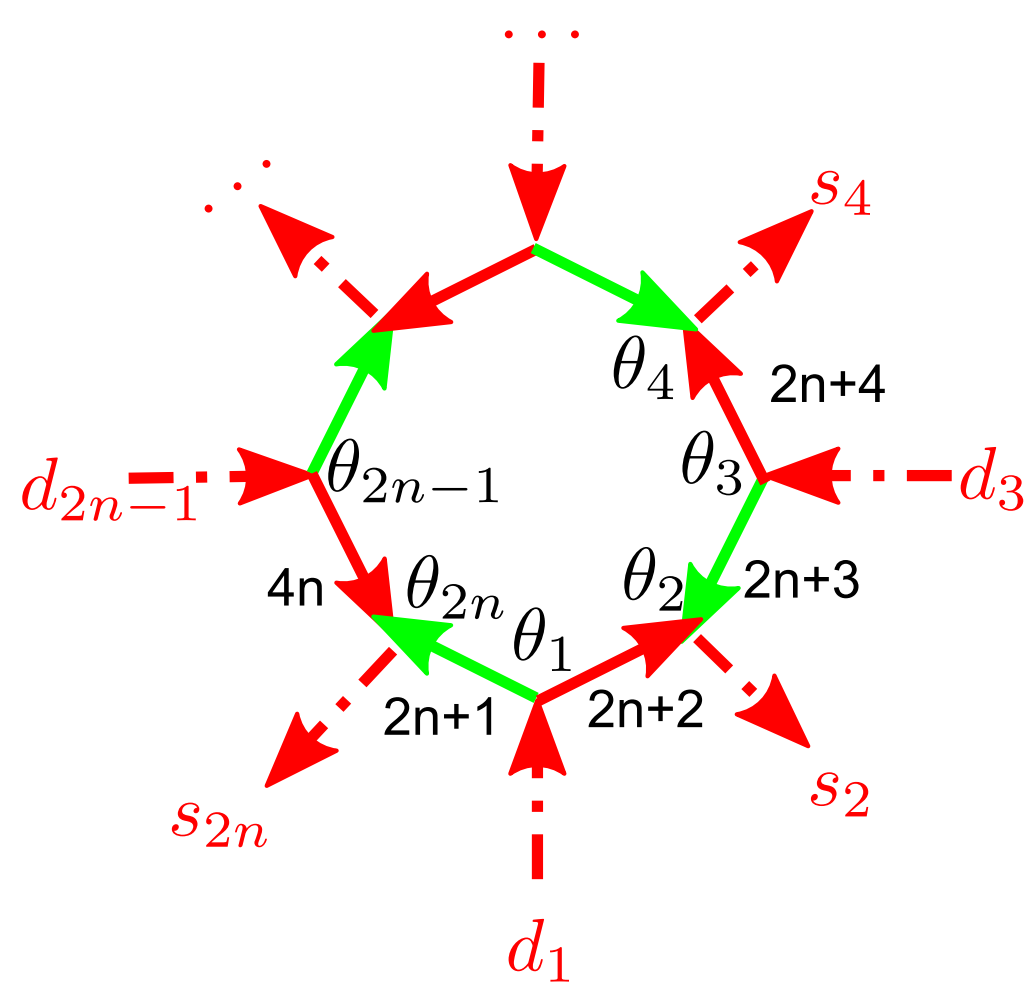

Figure 3: A diverge-merge network: at junction $j$, red links belong to $I_{j}^{+}$or $O_{j}^{+}$, and green links belong to $I_{j}^{-}$or $O_{j}^{-}$

a diverge junction, upstream to a destination is a merge junction, and vehicles between a pair of origin and destination always travel through first a diverge and then a merge.

Here we consider the stationary state when all origin and even-numbered regular links are SOC, all destination links are either SOC or C, and odd-numbered regular links are SUC. From Lemma 2.1, (10), and (9a), the sufficient and necessary conditions for links $2 n+1$ and $2 n+2$ to be stationary at SUC and SOC, 4 respectively, include $\theta_{2 n}^{*}<1$, and

$$
\begin{aligned}
& d_{2 n+2}^{-}=\min \left\{d_{1}, \frac{C_{2 n+1}}{1-\xi_{1}}\right\} \cdot \xi_{1}>s_{2 n+2}^{+}=C_{2 n+2} \theta_{2}^{*}, \\
& d_{2 n+1}^{-}=\min \left\{d_{1}, \frac{C_{2 n+2} \theta_{2}^{*}}{\xi_{1}}\right\} \cdot\left(1-\xi_{1}\right)<s_{2 n+1}^{+}=C_{2 n+1} \theta_{2 n}^{*} \leq C_{2 n+1},
\end{aligned}
$$

where $\theta_{2}^{*}$ and $\theta_{2 n}^{*}$ are stationary critical demand levels. If we assume that $d_{1}$ is sufficiently large, then the conditions are equivalent to

$$
\frac{1-\xi_{1}}{\xi_{1}}<\frac{C_{2 n+1} \theta_{2 n}^{*}}{C_{2 n+2} \theta_{2}^{*}}
$$

\footnotetext{
${ }^{4}$ Note that here SUC/SOC/ZS stationary states when $d_{a}^{-}=s_{a}^{+}$are considered neither SUC nor SOC.
} 
Similarly, at diverge $r(r=3,5, \cdots, 2 n-1)$ we have the following sufficient and necessary conditions for the traffic patterns shown in Figure 3

$$
\frac{1-\xi_{r}}{\xi_{r}}<\frac{C_{2 n+r} \theta_{r-1}^{*}}{C_{2 n+r+1} \theta_{r+1}^{*}}
$$

When (16) is satisfied, all junctions are SOC in stationary states. Then from Theorem 3.3 , we can see that information propagates in the clockwise direction, and (8) can be simplified as the following linear map

$$
\overrightarrow{\theta^{\prime}}=P \vec{\theta}+Q
$$

where $\vec{\theta}=\left[\theta_{1}, \theta_{2}, \cdots, \theta_{2 n-1}, \theta_{2 n}\right]^{T}$, and

$$
P=\left[\begin{array}{lllll}
0 & \frac{C_{2 n+2}}{C_{1} \xi_{1}} & & \cdots & \\
& 0 & -\frac{C_{3}\left(1-\xi_{3}\right)}{C_{2 n+2}} & & \\
\vdots & & \ddots & \ddots & \\
& \cdots & 0 & \frac{C_{4 n}}{C_{2 n-1} \xi_{2 n-1}} \\
-\frac{C_{1}\left(1-\xi_{1}\right)}{C_{4 n}} & & \cdots & & 0
\end{array}\right], \quad Q=\left[\begin{array}{c}
0 \\
\frac{s_{2}}{C_{2 n+2}} \\
\vdots \\
0 \\
\frac{s_{2 n}}{C_{4 n}}
\end{array}\right] .
$$

As in (13a), in the map, (17a), all critical demand levels are circularly dependent, corresponding to circular information propagation in the diverge-merge network. But note that the gridlock state for $\vec{\theta}=0$ is not a solution of (17a).

We observe that $P^{2 n}=(-1)^{n} \lambda I_{2 n \times 2 n}$, where $I_{2 n \times 2 n}$ is an identity matrix, and

$$
\lambda=\prod_{r \in R} \frac{1-\xi_{r}}{\xi_{r}} .
$$

Thus, instead of directly analyzing the $2 n$-dimensional map, (17a), we analyze the following equivalent one-dimensional map

$$
\theta_{1}^{(2 n)}=(-1)^{n} \lambda \theta_{1}+\epsilon_{1},
$$

where $\epsilon_{1}$ is the first element of $\sum_{i=0}^{2 n-1} P^{i} Q$. Then from (Galor, 2007, Chapter 1) we have the following observations regarding the new map (19):

1. When $\lambda>1$, there exists a unique fixed point, $\theta_{1}^{*}=\epsilon_{1} /\left(1+(-1)^{n} \lambda\right)$, and the fixed point is unstable.

2. When $\lambda=1, n$ is even, and $\epsilon_{1}=0$, there exist an infinite number of fixed points, and they are Lyapunov stable, but not asymptotically stable (LaSalle, 1976); when $\lambda=1$ and $n$ is odd, there exists one fixed point, $\theta_{1}^{*}=\epsilon_{1} / 2$, which is unstable. 
3. When $\lambda<1$, there exists a unique fixed point, $\theta_{1}^{*}=\epsilon_{1} /\left(1+(-1)^{n} \lambda\right)$, and the fixed point is asymptotically stable.

Furthermore, for a symmetric diverge-merge network with $C_{2 n+2 i-1}=2 C_{2 n+2 i}$ for $i=$ $1, \cdots, n$ and $\theta_{2}^{*}=\theta_{4}^{*}=\cdots=\theta_{2 n}^{*}$ in stationary states, we denote $\xi_{r}=\xi$ for all $r \in R$. From (16), we can see that the sufficient and necessary condition for a beltway network to be in the state shown in Figure 3 is equivalent to $\xi>\frac{1}{3}$. Further from (18) we have $\lambda=\left(\frac{1-\xi}{\xi}\right)^{n}$. Thus if $\frac{1}{3}<\xi<\frac{1}{2}$, the fixed point is unstable; if $\xi=\frac{1}{2}$, the fixed point is Lyapunov stable for an even $n$ and unstable for an odd $n$; if $\xi>\frac{1}{2}$, it is asymptotically stable. These conclusions are the same as those obtained in (Jin, 2013) through the Poincaré map approach. Thus for the diverge-merge network, the new map in critical demand levels has the same stability property as the Poincaré map but is more general.

\subsection{A special diverge-merge network with $n=1$}

When $n=1$, the diverge-merge network in Figure 3 becomes the one shown in Figure 1 in (Jin, 2013), which has been thoroughly studied. In this case, we denote $\xi_{1}=\xi$, and from (16) the sufficient and necessary condition for links 3 and 4 to be stationary at SUC and SOC, respectively, is given by

$$
\frac{1-\xi}{\xi}<\frac{C_{3}}{C_{4}}
$$

Furthermore, (17a) can be explicitly written as

$$
\begin{aligned}
\theta_{1}^{\prime} & =\frac{C_{4}}{C_{1} \xi} \theta_{2}, \\
\theta_{2}^{\prime} & =-\frac{C_{1}(1-\xi)}{C_{4}} \theta_{1}+\frac{s_{2}}{C_{4}},
\end{aligned}
$$

whose fixed points are $\theta_{1}^{*}=\frac{s_{2}}{C_{1}}$ and $\theta_{2}^{*}=\frac{s_{2} \xi}{C_{4}}$. If we denote $\tilde{\theta}_{1}=\theta_{1}-\theta_{1}^{*}$ and $\tilde{\theta}_{2}=\theta_{2}-\theta_{2}^{*}$, then (20) is equivalent to

$$
\begin{aligned}
& \tilde{\theta}_{1}^{\prime}=\frac{C_{4}}{C_{1} \xi} \tilde{\theta}_{2} \\
& \tilde{\theta}_{2}^{\prime}=-\frac{C_{1}(1-\xi)}{C_{4}} \tilde{\theta}_{1},
\end{aligned}
$$

whose characteristic equation is $y^{2}+\frac{1-\xi}{\xi}=0$, and the eigenvalues are imaginary numbers: $y= \pm \sqrt{\frac{1-\xi}{\xi}} i$. From (Galor, 2007), we can see that (20) is asymptotically stable if and only if $\frac{1-\xi}{\xi}<1$; i.e., if $\xi>\frac{1}{2}$. When $\xi \leq \frac{1}{2}$, it is unstable. The results are the same as those obtained from the one-dimensional map, (19). 
Combining the two equations in (20), we obtain a new map with respect to $\theta_{1}$ :

$$
\theta_{1}^{\prime \prime}=-\frac{1-\xi}{\xi} \theta_{1}+\frac{s_{2}}{C_{1} \xi}
$$

We denote the in-flux of link 3 by $f_{3}$. Then $f_{3}=C_{1} \xi \theta_{1}$, and

$$
f_{3}^{\prime \prime}=-\frac{1-\xi}{\xi} f_{3}+s_{2}
$$

which is equivalent to the Poincaré map derived in (Jin, 2013).

Similarly for general linear maps, (13a) and (17a), we can also derive their equivalent Poincaré maps in boundary fluxes. Note that, however, the Poincaré map can only be derived for specific networks with circular information propagation, including diverge-merge and beltway networks, but the new map in critical demand levels can be applied to find stationary states and analyze their stability property in general road networks.

\section{$5 \quad$ Stability of general stationary states}

In this section, we further apply the map to study general stationary states in a $2 \times 2$ grid network shown in Figure 4, in which there may not exist circular information propagation. Here dash-dotted lines are for the four origin links $(R=\{1,2,3,4\})$ and the four destination links $(W=\{5,6,7,8\})$, solid lines for the four regular links $(A=\{9,10,11,12\})$, and shaded disks for the four junctions $(J=\{1,2,3,4\})$. In this network, there are totally 16 commodities, since there exists one route between each origin-destination pair, and the commodity from origin $r$ to destination $w$ is labeled by $4 \cdot(r-1)+w-4$ for $r=1, \cdots, 4$ and $w=5, \cdots, 8$. There are four commodities on each link. We denote the matrix of route choice proportions by $\boldsymbol{\xi}$, where the $(r, w-4)$ th element is the route choice proportion between origin $r$ and destination $w$; thus the sum of each row equals 1 .

Here we assume that links 1, 3, 10, and 12 have the same capacity of 2, and all other links have the same capacity of 1 . We set the demands of origins 1 to 4 to be $1.8,1$, 2 , and 0.9 , respectively, the supplies of destinations 5 to 8 to be $0.8,1,0.9$, and 0.9 , respectively, and the route choice proportions to be

$$
\boldsymbol{\xi}=\begin{gathered}
5 \\
1 \\
2 \\
3 \\
4
\end{gathered}\left[\begin{array}{cccc}
0.25 & 0.25 & 0.3 & 8 \\
0.2 & 0.3 & 0.4 & 0.1 \\
0.25 & 0.2 & 0.3 & 0.25 \\
0.3 & 0.25 & 0.15 & 0.3
\end{array}\right]
$$




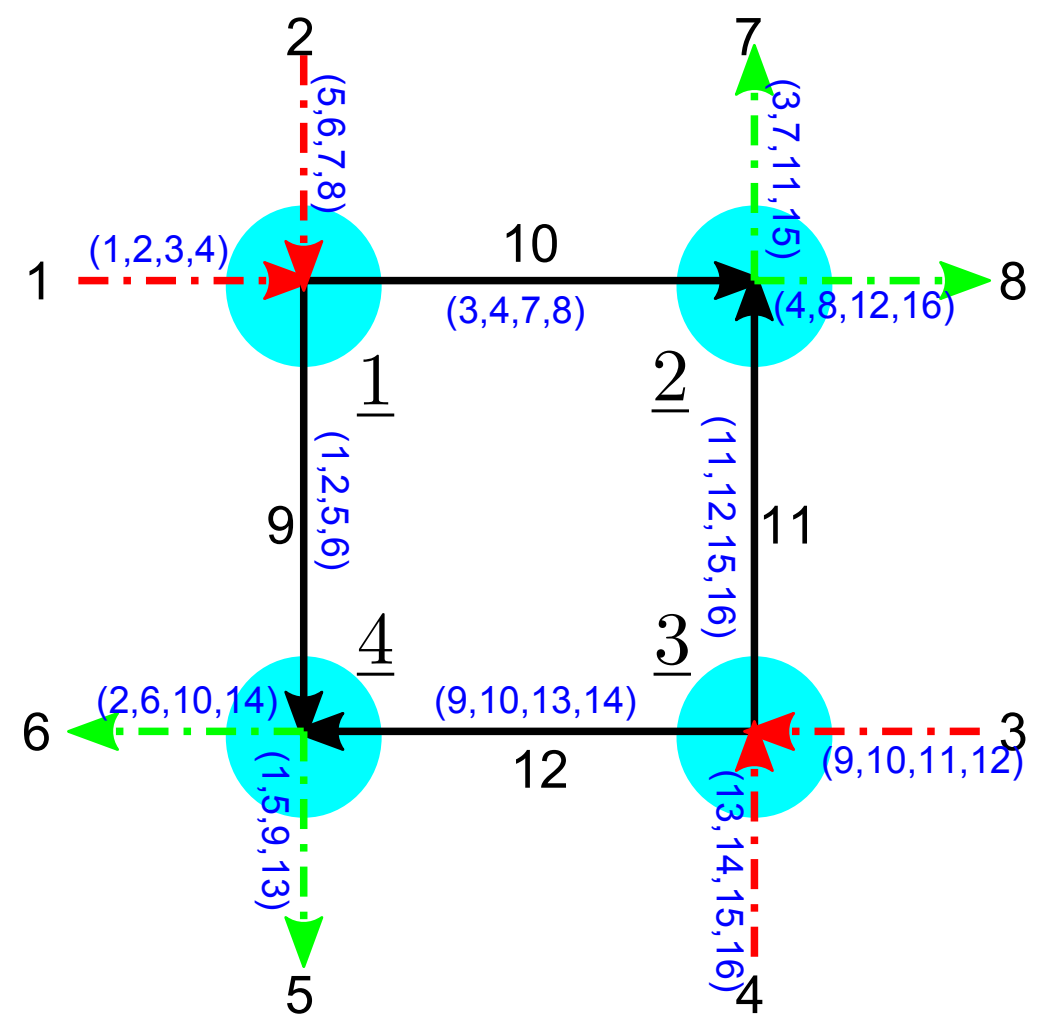

Figure 4: A $2 \times 2$ grid network: blue numbers in parentheses are for commodities

which leads to the following turning proportions at junctions 1 and 3 :

$$
\begin{aligned}
\xi_{1 \rightarrow 10} & =\xi_{1,7}+\xi_{1,8}=0.5, \\
\xi_{2 \rightarrow 10} & =\xi_{2,7}+\xi_{2,8}=0.5, \\
\xi_{1 \rightarrow 9} & =1-\xi_{1 \rightarrow 10}=0.5 \\
\xi_{2 \rightarrow 9} & =1-\xi_{2 \rightarrow 10}=0.5 \\
\xi_{3 \rightarrow 11} & =\xi_{3,7}+\xi_{3,8}=0.55 \\
\xi_{4 \rightarrow 11} & =\xi_{4,7}+\xi_{4,8}=0.45 \\
\xi_{3 \rightarrow 12} & =1-\xi_{3 \rightarrow 11}=0.45 \\
\xi_{4 \rightarrow 12} & =1-\xi_{4 \rightarrow 11}=0.55 .
\end{aligned}
$$

In such a network, there may not exist circular information propagation, and we first study fixed points and their stability property with the map in critical demand levels, (8), and then verify the results with the Cell Transmission Model simulations (Daganzo, 1995) with the general junction model in (4). 


\subsection{Fixed points and their stability}

With different initial critical demand levels, we find two fixed points for (8), in which all origin links are SOC. Thus in stationary states, $q_{1}=2 \theta_{1}, q_{2}=\theta_{1}, q_{3}=2 \theta_{3}, q_{4}=\theta_{3}$, $q_{5}=\left(2 \xi_{1,5}+\xi_{2,5}\right) \theta_{1}+\left(2 \xi_{3,5}+\xi_{4,5}\right) \theta_{3}=0.7 \theta_{1}+0.8 \theta_{3}, q_{6}=0.8 \theta_{1}+0.65 \theta_{3}, q_{7}=\theta_{1}+0.75 \theta_{3}$, $q_{8}=0.5 \theta_{1}+0.8 \theta_{3}, q_{9}=\left(2 \xi_{1 \rightarrow 9}+\xi_{2 \rightarrow 9}\right) \theta_{1}=1.5 \theta_{1}, q_{10}=\left(2 \xi_{1 \rightarrow 10}+\xi_{2 \rightarrow 10}\right) \theta_{1}=1.5 \theta_{1}$, $q_{11}=\left(2 \xi_{3 \rightarrow 11}+\xi_{4 \rightarrow 11}\right) \theta_{3}=1.55 \theta_{3}$, and $q_{12}=\left(2 \xi_{3 \rightarrow 12}+\xi_{4 \rightarrow 12}\right) \theta_{3}=1.45 \theta_{3}$. Furthermore, the turning proportions at junctions 2 and 4 can be calculated:

$$
\begin{aligned}
\xi_{10 \rightarrow 7} & =\frac{2}{3}, \quad \xi_{10 \rightarrow 8}=\frac{1}{3}, \\
\xi_{11 \rightarrow 7} & =\frac{0.75}{1.55}, \quad \xi_{11 \rightarrow 8}=\frac{0.8}{1.55}, \\
\xi_{9 \rightarrow 5} & =\frac{0.7}{1.5}, \quad \xi_{9 \rightarrow 6}=\frac{0.8}{1.5}, \\
\xi_{12 \rightarrow 5} & =\frac{0.8}{1.45}, \quad \xi_{12 \rightarrow 6}=\frac{0.65}{1.45} .
\end{aligned}
$$
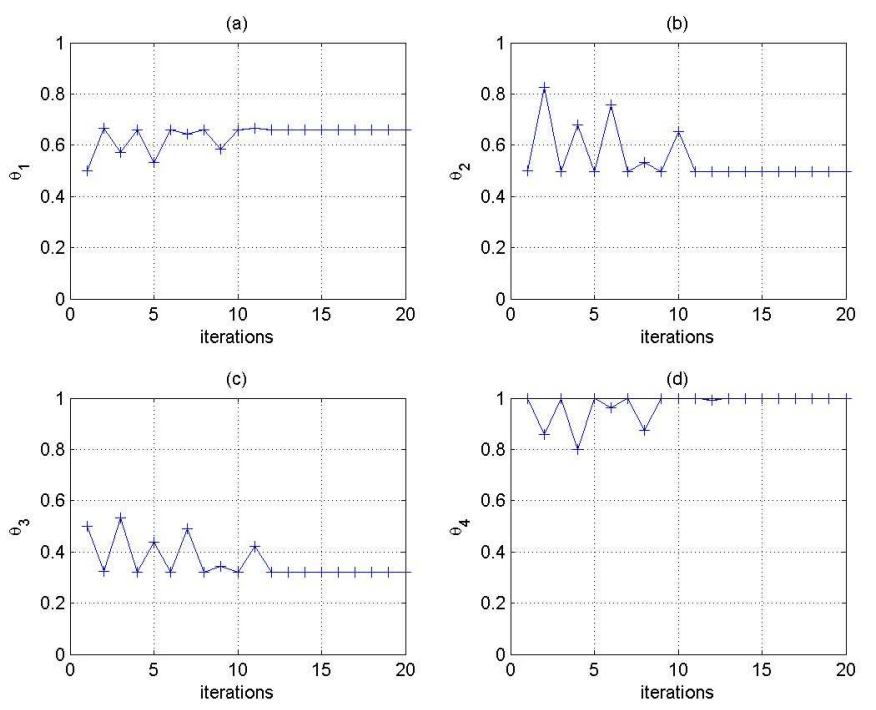

Figure 5: First 20 iterations of the critical demand levels of a stable fixed point

In Figure 5, we plot the first 20 iterations of the critical demand levels with the initial critical demand levels $\vec{\theta}=[0.5,0.5,0.5,1]^{T}$. We can see that the map, (8), converges within a finite number of steps to a stable fixed point, $\vec{\theta}^{*}=[0.6604,0.4953,0.3195,1]^{T}$. Following Lemma 2.1, we can determine the criticality of all regular links as follows: link 9 is stationary at SUC, since $q_{9}=1.5 \theta_{1}<s_{9}^{+}=C_{9} \theta_{4}$; link 10 is stationary at SOC, since $q_{10}=1.5 \theta_{1}=$ 
$s_{10}^{+}=C_{10} \theta_{2}$; link 11 is stationary at SOC, since $q_{11}=1.55 \theta_{3}=s_{11}^{+}=C_{11} \theta_{2}$; link 12 is stationary at SUC, since $q_{12}=1.45 \theta_{3}<s_{12}^{+}=C_{12} \theta_{4}$; link 7 is stationary at SOC, since $q_{7}=\theta_{1}+0.75 \theta_{3}=s_{7}<C_{7}$; and all other destination links are stationary at SUC. In this case, junctions 1, 2, and 3 are SOC, but junction 4 is SUC, and there exists no circular information propagation. But after knowing the criticality of all links, we can obtain the following simplified linear map from (9h):

$$
\begin{aligned}
\theta_{1}^{\prime} & =\frac{2 \theta_{2}}{2 \xi_{1 \rightarrow 10}+\xi_{2 \rightarrow 10}}=\frac{2 \theta_{2}}{1.5}=0.6604 \\
\theta_{2}^{\prime} & =\frac{s_{7}}{2 \xi_{10 \rightarrow 7}+\xi_{11 \rightarrow 7}}=\frac{s_{7}}{\frac{4}{3}+\frac{0.75}{1.55}}=0.4953 \\
\theta_{3}^{\prime} & =\frac{\theta_{2}}{2 \xi_{3 \rightarrow 11}+\xi_{4 \rightarrow 11}}=\frac{\theta_{2}}{1.55}=0.3195 \\
\theta_{4}^{\prime} & =1
\end{aligned}
$$

from which we can verify that $[0.6604,0.4953,0.3195,1]^{T}$ is indeed a stable fixed point, and the map can converge to the fixed point in a finite number of steps.
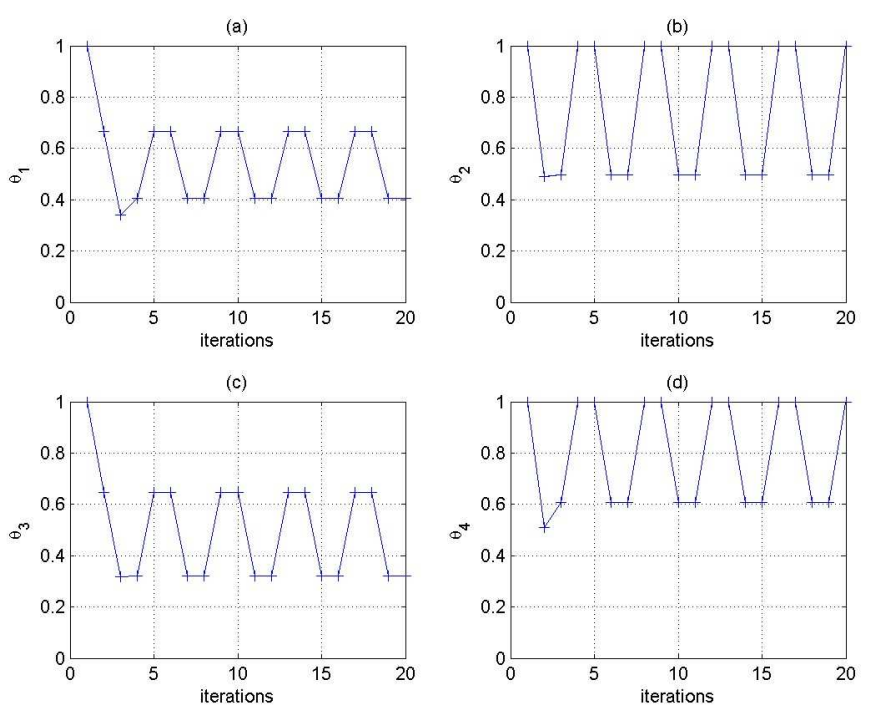

Figure 6: First 20 iterations of the critical demand levels of an unstable fixed point

In Figure 6, we plot the first 20 iterations of the critical demand levels with the initial critical demand levels $\vec{\theta}=[1,1,1,1]^{T}$. We can see that the map, (8) , develops a periodical pattern of period 4 . In this case, the fixed point cannot be directly calculated from the map. Instead, we first determine through trial and error the criticality of all links: links 5 and 7 are stationary at SOC, links 6 and 8 at SUC, links 9 and 11 at SOC, and links 10 and 12 at 
SUC. Then we can obtain the following simplified linear map from (9h):

$$
\begin{aligned}
\theta_{1}^{\prime} & =\frac{\theta_{4}}{2 \xi_{1 \rightarrow 9}+\xi_{2 \rightarrow 9}}=\frac{\theta_{4}}{1.5}, \\
\theta_{2}^{\prime} & =\frac{s_{7}-q_{10} \xi_{10 \rightarrow 7}}{\xi_{11 \rightarrow 7}}=\frac{0.9-\theta_{1}}{0.75 / 1.55}, \\
\theta_{3}^{\prime} & =\frac{\theta_{2}}{2 \xi_{3 \rightarrow 11}+\xi_{4 \rightarrow 11}}=\frac{\theta_{2}}{1.55}, \\
\theta_{4}^{\prime} & =\frac{s_{5}-q_{12} \xi_{12 \rightarrow 5}}{\xi_{9 \rightarrow 5}}=\frac{0.8-0.8 \theta_{3}}{0.7 / 1.5},
\end{aligned}
$$

in which the critical demand levels are circularly dependent, and there exists clockwise circular information propagation on the four regular links in stationary states. Thus we can obtain the following one-dimensional map

$$
\theta_{4}^{(4)}=\frac{32}{21} \theta_{4}-\frac{12}{35}
$$

from which we can find the following fixed point: $\left[\frac{24}{55}, \frac{527}{550}, \frac{34}{55}, \frac{36}{55}\right]^{T}$. We can verify that the map, (8), stays at this fixed point if it is set as the initial condition. Further from the one-dimensional map, we can see that the fixed point is unstable.

Comparing the two stationary states, we have the following observations:

1. Once we know the criticality of all links in a stationary state, the original map, (8), can be simplified into a linear map, from which the fixed point can be calculated and its stability analyzed. Thus, the map in critical demand levels can be applied to analyze stability of general stationary states.

2. The existence of the second unstable fixed point confirms that a necessary condition for a stationary state in the grid network to be unstable is indeed the existence of circular information propagation in the state and circular dependence among critical demand levels in the simplified linear map of (8). Note that, however, circular information propagation may not be sufficient for the existence of unstable stationary states.

3. In the stable stationary state, only one destination link 7 is SOC, and its supply is completely consumed. In the unstable stationary state, both links 5 and 7 are SOC, and their supplies are completely consumed. Furthermore, the total stationary flowrate of the network in stationary states equals $3 \theta_{1}+3 \theta_{3}$, which is 2.9397 for the stable stationary state and 3.1636 for the unstable one. In this sense, the unstable stationary state is more efficient than the stable stationary state.

\subsection{Verification with the Cell Transmission Model}

In this subsection, we study the stationary states and their stability property for the grid network in Figure 4 with the Cell Transmission Model (Daganzo, 1995), which is the discrete 
version of the network kinematic wave model presented in Section 2.1. Here all links have the same length of 1 with a coordinate $x \in[0,1]$ which increases in the traffic direction. We denote the densities of four commodities on a regular link $a$ by $k_{a, \omega}(x, t)$, where $\omega \in \Omega_{a}$. Thus the total density $k_{a}(x, t)=\sum_{\omega \in \Omega_{a}} k_{a, \omega}(x, t)$.

We apply the following triangular fundamental diagram for all links (Munjal et al., 1971; Haberman, 1977; Newell, 1993):

$$
Q_{a}\left(k_{a}\right)=\min \left\{k_{a}, \frac{1}{4}\left(5 C_{a}-k_{a}\right)\right\},
$$

where the free-flow speed is $v_{f}=1$, the link capacity $C_{a}$, and the jam density $5 C_{a}$. The corresponding demand and supply functions are given by (Daganzo, 1995; Lebacque, 1996):

$$
\begin{aligned}
D_{a}\left(k_{a}\right) & =\min \left\{k_{a}, C_{a}\right\}, \\
S_{a}\left(k_{a}\right) & =\min \left\{\frac{1}{4}\left(5 C_{a}-k_{a}\right), C_{a}\right\} .
\end{aligned}
$$

In addition, the simulation time-step size is $\Delta t=\frac{1}{N}$, and each link is divided into $N$ cells with the cell size of $\Delta x=\frac{1}{N}$. Thus the CFL number is $v_{f} \frac{\Delta t}{\Delta x}=1$. We denote the center of cell $i(i=1, \cdots, N)$ by $x_{i}$, and the average total and commodity densities on link $a$ at time step $l$ by $k_{a, i}^{l}$, and $k_{a, \omega, i}^{l}$, respectively. Then based on the conservation of traffic flow, (1a) and (1c), we can update traffic densities as follows:

$$
\begin{aligned}
k_{a, i}^{l+1} & =k_{a, i}^{l}+\frac{\Delta t}{\Delta x}\left(f_{a, i}^{l}-g_{a, i}^{l}\right), \\
k_{a, \omega, i}^{l+1} & =k_{a, \omega, i}^{l}+\frac{\Delta t}{\Delta x}\left(\phi_{a, \omega, i}^{l}-\psi_{a, \omega, i}^{l}\right),
\end{aligned}
$$

where $f_{a, i}^{l}$ and $g_{a, i}^{l}$ are total in- and out-fluxes from step $l$ to step $l+1$ for cell $i$, and $\phi_{a, \omega, i}^{l}$, and $\psi_{a, \omega, i}^{l}$ are the corresponding commodity in- and out-fluxes. The in- and out-fluxes for regular links are calculated as follows:

1. Inside link $a$, for $i=1, \cdots, N-1$, we assume that vehicles follow the first-in-first-out principle (Daganzo, 1995; Lebacque, 1996):

$$
\begin{aligned}
g_{a, i}^{l} & =f_{a, i+1}^{l}=\min \left\{D_{a}\left(k_{a, i}^{l}\right), S_{a}\left(k_{a, i+1}^{l}\right)\right\}, \\
\psi_{a, \omega, i}^{l} & =\phi_{a, \omega, i+1}^{l}=g_{a, i}^{l} \frac{k_{a, \omega, i}^{l}}{k_{a, i}^{l}} .
\end{aligned}
$$

2. The in- and out-fluxes at the four junctions are calculated from the junction model (41), which is not repeated here. 
For the second unstable fixed point discussed in the preceding subsection, the corresponding stationary traffic densities are given by:

$$
\begin{aligned}
k_{9, i}^{0} & =5 C_{9}-4 q_{9}=5 C_{9}-6 \theta_{1}^{*}, \\
k_{10, i}^{0} & =q_{10}=1.5 \theta_{1}^{*}, \\
k_{11, i}^{0} & =5 C_{11}-4 q_{11}=5 C_{11}-6.2 \theta_{3}^{*}, \\
k_{12, i}^{0} & =1.45 \theta_{3}^{*},
\end{aligned}
$$

where $\theta_{1}^{*}=\frac{24}{55}$ and $\theta_{3}^{*}=\frac{34}{55}$, and the commodity proportions are constant and can be determined by the route choice proportions. It can be verified that solutions of the Cell Transmission Model stay at the stationary states if we use these as initial densities.

However, if we apply a small disturbance to the first cell on link 9 such that $k_{9,1}^{0}=$ $0.9\left(5 C_{9}-6 \theta_{1}^{*}\right)$, the evolution of the four critical demand levels at the four junctions from 0 to 800 is shown in Figure 7 , and the evolution of densities on the four regular links is shown in Figure 8, where we normalize the densities by the respective jam densities on all links. Here we set $N=20$. From Figure 7 we can see that, initially, the critical demand levels are almost the same as those in the unstable fixed point, $\left[\frac{24}{55}, \frac{527}{550}, \frac{34}{55}, \frac{36}{55}\right]^{T}$, but the small disturbance drives the system away from the unstable fixed point until it reaches the stable fixed point, $[0.6604,0.4953,0.3195,1]^{T}$. From Figure 8 , we have the following observations: (i) Initially link 9 is congested with a normalized density greater than 0.2 as in the unstable stationary state, but eventually it becomes uncongested with a normalized density smaller than 0.2. (ii) Link 10 is initially uncongested, but its density keeps increasing, until at around $t=160$, when a major shock wave forms on the link and the link becomes congested. This sudden change can also be confirmed in Figure 7. (iii) Link 11 is initially congested but gets more congested eventually. (iv) Link 12 is initially uncongested and becomes more so eventually. These results further confirm that the stability of the fixed points for the map, (8), is consistent with the stability of the corresponding stationary states.

\section{Conclusion}

In this study, we presented a new method to determine the stability property of stationary states in a general road network based on a discrete map in critical demand levels, which was proposed in (Jin, 2015) to prove the existence of stationary states. We first reviewed a network kinematic wave model and properties of stationary states on a link and then defined the criticality of stationary junctions. After presenting the map in critical demand levels, we discussed information propagation in the map regarding the inter-dependence of critical demand levels. For beltway and diverge-merge networks with circular information propagation, we analytically derived the stability conditions for stationary states and demonstrated that they are consistent with those obtained through the Poincaré map approach in (Jin, 2013 ) but more general. Further for a $2 \times 2$ grid network, from the map in critical demand 

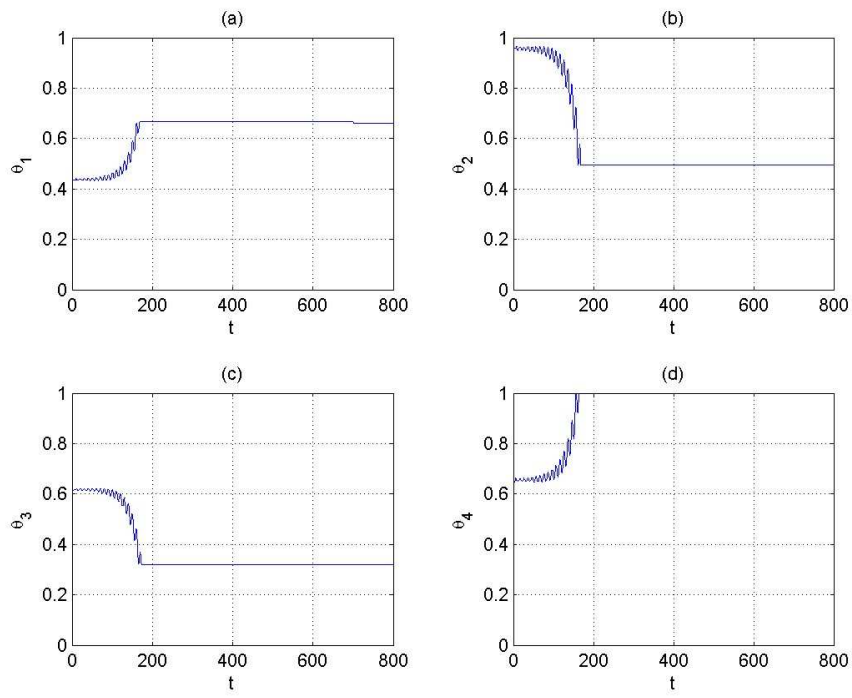

Figure 7: Evolution of the critical demand levels in the Cell Transmission Model

levels we found two stationary states, one stable, and one unstable, and verified the results with the Cell Transmission Model simulations. We therefore conclude that the stability of fixed points for the new map in critical demand levels is indeed the same as the stability of stationary states, and that the new approach is more general than the Poincaré map approach, since it can be applied to general stationary states without circular information propagation.

In Section 4, for the beltway and diverge-merge networks, we showed that circular information propagation can lead to unstable stationary states. In Section 5, for the grid network, we showed that there exists circular information propagation in the unstable stationary state. It suggests that circular information propagation is the necessary condition for the existence of unstable stationary states in general road networks. But a rigorous proof of this statement is yet to be obtained.

In Sections 4 and 5, we found that, once the criticality of each link in a stationary state is known, we can always derive a simplified linear map in critical demand levels, with which we can then calculate the fixed point and analyze its stability. However, a systematic method to derive such a linear map is subject to further studies. This will also help to find all stationary states in a network.

Also for the $2 \times 2$ grid network we found that there can be two stationary states: one stable, and the other unstable. However, the unstable one is more efficient, in the sense that its total flow-rate is higher. In the future we will also be interested in studying the efficiency of stationary states in a general road networks.

As shown in Section 4 all intersections in the beltway network or the diverge-merge 

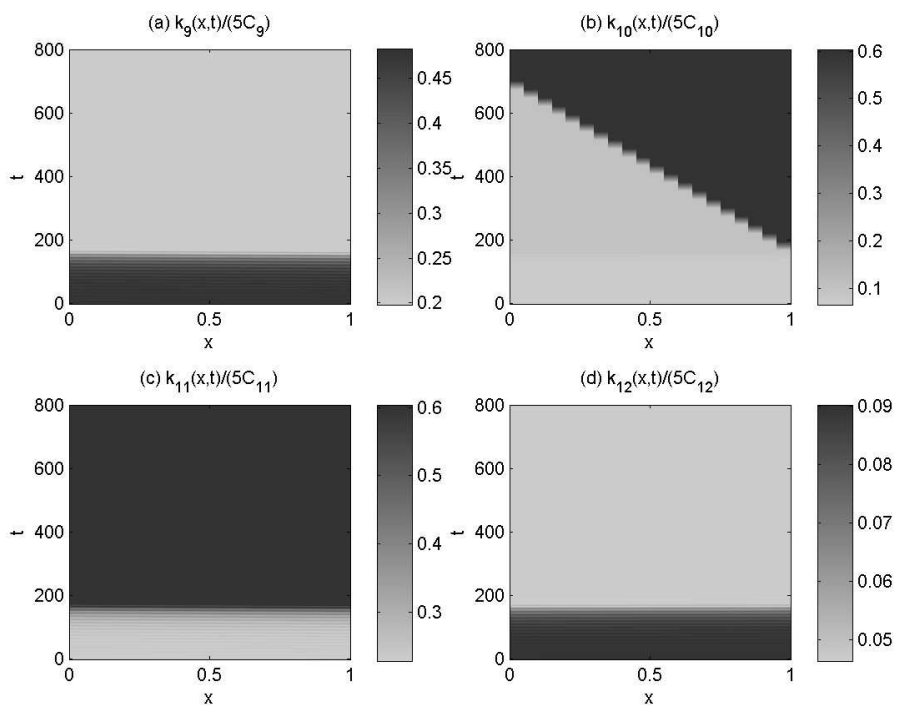

Figure 8: Contour plots of densities on four regular links normalized by the respective jam densities

network are inter-dependent, and information propagates in the whole road network. Thus it is generally not possible to decouple a network into sub-networks. That said, however, it might be possible for some specific networks, where traffic conditions in several sub-networks are independent of each other. In the future we will be interested in determining independent sub-networks for a general road network with the help of the discrete map in critical demand levels.

In the future, we will be interested in studying the properties of stationary states in road networks with junction models different from (44). In particular we will attempt to establish a discrete map similar to (8), if a given junction model is invariant, and the critical demand level is well-defined.

\section{Appendix A. Proof of Lemma 2.3}

Proof. Here we prove the first statement by contradiction. If $O_{j}^{+}$is empty, then all downstream links are $\mathrm{SUC}$ or $\mathrm{ZS}$, and $q_{b}<s_{b}=C_{b}$ for $b \in O_{j}$. Assuming that there exists a nonempty set of SOC or ZS upstream links, $I_{j}^{+} \subseteq I_{j}$. Then $\frac{d_{a}}{C_{a}}=1$ for $a \in I_{j}^{+}$, and $1>\theta_{j} \geq \frac{d_{\alpha}}{C_{\alpha}}$ for $\alpha \in I_{j}^{-}$. From (4e) and (4g), we have that for all $b \in O_{j}$

$$
q_{b}=\sum_{\alpha \in I_{j}^{-}} d_{\alpha} \xi_{\alpha \rightarrow b}+\sum_{a \in I_{j}^{+}} C_{a} \theta_{j} \xi_{a \rightarrow b}<s_{b}=C_{b} .
$$


Thus from (4b) we have

$$
\gamma_{b}\left(I_{j}^{+}\right)=\frac{s_{b}-\sum_{\alpha \in I_{j}^{-}} d_{\alpha} \xi_{\alpha \rightarrow b}}{\sum_{a \in I_{j}^{+}} C_{a} \xi_{a \rightarrow b}}>\theta_{j} .
$$

From (4c) and (4d), we have $\theta_{j}=\min \left\{1, \max _{A \neq \emptyset, A \subseteq I_{j}} \gamma_{b}(A)\right\} \geq \gamma_{b}\left(I_{j}^{+}\right)>\theta_{j}$, which is impossible. Thus $I_{j}^{+}$has to be empty.

\section{Appendix B. Proof of Lemma 2.6}

Proof. From (4g), we have $q_{b}=\sum_{a \in I_{j}} q_{a} \xi_{a \rightarrow b}$. Thus $s_{b}=q_{b}=\sum_{a \in I_{j}} q_{a} \xi_{a \rightarrow b} \leq \sum_{a \in I_{j}} d_{a} \xi_{a \rightarrow b}$, which leads to $\pi_{b}=s_{b}-\sum_{a \in I_{j}} d_{a} \xi_{a \rightarrow b} \leq 0$. Since $O_{j}^{+}$is not empty, the junction can be stationary at $\mathrm{C}$ or SOC.

1. If $\pi_{b}=0$, then $q_{a}=d_{a}$ for all $a \in I_{j}$. In this case, $I_{j}^{+}$is empty, and the junction is stationary at C. From (4e) we then have $\theta_{j} \geq \mu_{a}$ for all $a \in I_{j}$; hence, $\theta_{j} \geq \max _{a \in I_{j}} \mu_{a}$, which leads to $\min _{\beta \in O_{j}} \Gamma_{\beta} \geq \max _{a \in I_{j}} \mu_{a}$ from (4d). Since $\pi_{b}=0, \Gamma_{b}=\max _{a \in I_{j}} \mu_{a}$ from Lemma 2.4. Thus $\theta_{j}=\min _{\beta \in O_{j}} \Gamma_{\beta}=\Gamma_{b}=\max _{a \in I_{j}} \mu_{a}$.

2. If $\pi_{b}<0$, then $\min _{\beta \in O_{j}} \pi_{\beta}<0$. From Lemma 2.5 there exists a unique non-empty $A_{*} \subseteq I_{j}$ such that

$$
\min _{a \in A_{*}} \mu_{a}>\min _{\beta \in O_{j}} \Gamma_{\beta} \geq \max _{a \in I_{j} \backslash A_{*}} \mu_{a}
$$

In this case, $O_{j}^{+}$is not empty, and the junction is stationary at SOC. From (4d) we have $\theta_{j}=\min _{\beta \in O_{j}} \Gamma_{\beta}$. From (4e), $q_{a}=d_{a}$ for $a \in I_{j} \backslash A_{*}$, and $q_{a}=C_{a} \theta_{j}$ for $a \in A_{*}$. Thus from (4g) we have

$$
q_{b}=\sum_{a \in I_{j} \backslash A_{*}} d_{a} \xi_{a \rightarrow b}+\sum_{a \in A_{*}} C_{a} \theta_{j} \xi_{a \rightarrow b},
$$

which leads to

$$
s_{b}=\sum_{a \in I_{j} \backslash A_{*}} d_{a} \xi_{a \rightarrow b}+\sum_{a \in A_{*}} C_{a} \theta_{j} \xi_{a \rightarrow b}
$$

Thus from (4b),

$$
\gamma_{b}\left(A_{*}\right)=\frac{\pi_{b}+\sum_{a \in A_{*}} d_{a} \xi_{a \rightarrow b}}{\sum_{a \in A_{*}} C_{a} \xi_{a \rightarrow b}}=\theta_{j} .
$$

Since $\pi_{b}<0$ and $A_{*}$ is the unique nonempty set such that $\mu_{a}>\gamma_{b}\left(A_{*}\right) \geq \mu_{\alpha}$ for $a \in A_{*}$ and $\alpha \in I_{j} \backslash A_{*}, \Gamma_{b}=\gamma_{b}\left(A_{*}\right)$ from Lemma 2.4. Therefore, $I_{j}^{+}=A_{*}$, and $\theta_{j}=\Gamma_{b}$. 


\section{Appendix C. Proof of Theorem 2.7}

Proof. From Lemma 2.5, $\min _{b \in O_{j}} \pi_{b}>,=,<0$ if and only if $\min _{b \in O_{j}} \Gamma_{b}>,=,<\max _{a \in I_{j}} \frac{d_{a}}{C_{a}}$ respectively.

We first prove that a junction is SUC if and only if $\min _{b \in O_{j}} \pi_{b}>0$.

- If a junction is SUC, then both $I_{j}^{+}$and $O_{j}^{+}$are empty. Thus $d_{a}=q_{a} \leq C_{a}$ for any $a \in I_{j}$ and $q_{b}<s_{b}=C_{b}$ for any $b \in O_{j}$. From (4g) we have $q_{b}=\sum_{a \in I_{j}} d_{a} \xi_{a \rightarrow b}<s_{b}$ for any $b \in O_{j}$. Thus $\min _{b \in O_{j}} \pi_{b}>0$.

- If $\min _{b \in O_{j}} \pi_{b}>0$, then from Lemma 2.5 we have $\min _{b \in O_{j}} \Gamma_{b}>\max _{a \in I_{j}} \frac{d_{a}}{C_{a}}$. Thus $\theta_{j} \geq \max _{a \in I_{j}} \frac{d_{a}}{C_{a}}$. From (4e) we have $q_{a}=d_{a} \leq C_{a}$ for any $a \in I_{j}$, and all upstream links are SUC or C. From (4g) we have $q_{b}=\sum_{a \in I_{j}} d_{a} \xi_{a \rightarrow b}<s_{b}$ since $\pi_{b}>0$ for any $b \in O_{j}$, and all downstream links are SUC or ZS. Thus the junction is SUC.

We then prove that a junction is $\mathrm{C}$ if and only if $\min _{b \in O_{j}} \pi_{b}=0$.

- If a junction is $\mathrm{C}$, then $I_{j}^{+}$is empty, but $O_{j}^{+}$not. Thus $d_{a}=q_{a} \leq C_{a}$ for any $a \in I_{j}$ and $q_{\beta}<s_{\beta}=C_{\beta}$ for any $\beta \in O_{j}^{-}$. For $b \in O_{j}^{+}$, from Lemma 2.6 we have $q_{b}=s_{b}$ and $\theta_{j}=\Gamma_{b}$. Thus from (4g) we have $q_{b}=\sum_{a \in I_{j}} d_{a} \xi_{a \rightarrow b}=s_{b}$ for $b \in O_{j}^{+}$, which leads to $\pi_{b}=0$ for $b \in O_{j}^{+}$. But for $\beta \in O_{j}^{-}, q_{\beta}=\sum_{a \in I_{j}} d_{a} \xi_{a \rightarrow \beta}<s_{\beta}$, which leads to $\pi_{\beta}>0$. Hence $\min _{b \in O_{j}} \pi_{b}=0$.

- If $\min _{b \in O_{j}} \pi_{b}=0$, then from Lemma 2.5 we have $\min _{b \in O_{j}} \Gamma_{b}=\max _{a \in I_{j}} \frac{d_{a}}{C_{a}} \leq 1$. Thus $\theta_{j}=\max _{a \in I_{j}} \frac{d_{a}}{C_{a}}$. From (4e) we have $q_{a}=d_{a} \leq C_{a}$ for any $a \in I_{j}$, and all upstream links are SUC or C. From (4g) we have $q_{b}=\sum_{a \in I_{j}} d_{a} \xi_{a \rightarrow b}$ for any $b \in O_{j}$. Since $\min _{b \in O_{j}} \pi_{b}=0$, there exists at least one $b$, such that $\pi_{b}=0$, for which $s_{b}=q_{b}$ and link $b$ is SOC or C. Thus $O_{j}^{+}$is not empty, and the junction is C.

We finally prove that a junction is SOC if and only if $\min _{b \in O_{j}} \pi_{b}<0$.

- If a junction is SOC, then both $I_{j}^{+}$and $O_{j}^{+}$are nonempty. Thus $q_{a}=C_{a} \theta_{j}<d_{a}=C_{a}$ for $a \in I_{j}^{+}$, and $d_{a}=q_{a} \leq C_{a}$ for $a \in I_{j}^{-}$. For $b \in O_{j}^{+}$, from Lemma 2.6 we have $q_{b}=s_{b}$ and $\theta_{j}=\Gamma_{b}$; from (4g) we have $q_{b}=\sum_{a \in I_{j}^{+}} C_{a} \theta_{j} \xi_{a \rightarrow b}+\sum_{a \in I_{j}^{-}} d_{a} \xi_{a \rightarrow b}<\sum_{a \in I_{j}} d_{a} \xi_{a \rightarrow b}$. Thus $\pi_{b}<0$ for $b \in O_{j}^{+}$, and $\min _{b \in O_{j}} \pi_{b}<0$.

- If $\min _{b \in O_{j}} \pi_{b}<0$, then from Lemma 2.5 there exists a unique nonempty $A_{*} \subseteq I_{j}$ such that

$$
\min _{a \in A_{*}} \mu_{a}>\min _{b \in O_{j}} \Gamma_{b} \geq \max _{a \in I_{j} \backslash A_{*}} \mu_{a}
$$

Thus $\min _{a \in A_{*}} \mu_{a}>\theta_{j}=\min _{b \in O_{j}} \Gamma_{b} \geq \max _{a \in I_{j} \backslash A_{*}} \mu_{a}$. Hence $I_{j}^{+}=A_{*}$ is nonempty, and the junction is SOC. 


\section{Appendix D. Proof of Theorem 3.3}

Proof. If link $a$ is SUC, then $a \in O_{j}^{-}$and $a \in I_{j+1}^{-}$. Thus from 4ge have

$$
q_{a}=\theta_{j} \sum_{\alpha \in I_{j}^{+}} C_{a} \xi_{\alpha \rightarrow a}+\sum_{\alpha \in I_{j}^{-}} d_{\alpha} \xi_{\alpha \rightarrow a} .
$$

Then from (9h), we have the map

$$
\theta_{j+1}^{\prime}=\frac{s_{b}-q_{a}-\sum_{\alpha \in I_{j+1}^{-}, \alpha \neq a} q_{\alpha} \xi_{\alpha \rightarrow b}}{\sum_{\alpha \in I_{j+1}^{+}} C_{\alpha} \xi_{\alpha \rightarrow b}}
$$

where $b \in O_{j+1}^{+}$. This leads to (11). We can see that in the map, (11), the downstream junction's critical demand level depends on and decreases with the upstream junction's, and information propagates forward along the link.

If link $a$ is SOC, then $a \in O_{j}^{+}$and $a \in I_{j+1}^{+}$. Thus from (4e) we have

$$
q_{a}=C_{a} \theta_{j+1}
$$

From (9h) and Lemma 2.6 we have

$$
\theta_{j}^{\prime}=\frac{q_{a}-\sum_{\alpha \in I_{j}^{-}} d_{\alpha} \xi_{\alpha \rightarrow a}}{\sum_{\alpha \in I_{j}^{+}} C_{\alpha} \xi_{\alpha \rightarrow a}}
$$

which leads to (12). We can see that in the map, (12), the upstream junction's critical demand level depends on and increases with the downstream junction's, and information propagates backward along the link.

\section{Acknowledgments}

We would like to thank three anonymous reviewers for their constructive and insightful comments and questions. This research is partially sponsored by NSF-CMMI: 1434753 .

\section{References}

Beckmann, M., McGuire, C. B., Winsten, C. B., 1956. Studies in the economics of transportation. Yale University Press, New Haven, Connecticut, also published as Rand-RM1488-PR, Rand Corporation, Santa Monica, CA, May 12, 1955.

Daganzo, C. F., 1995. The cell transmission model II: Network traffic. Transportation Research Part B 29 (2), 79-93. 
Daganzo, C. F., 1996. The nature of freeway gridlock and how to prevent it. Proceedings of the 13th International Symposium on Transportation and Traffic Theory, 629-646.

Daganzo, C. F., 2007. Urban gridlock: Macroscopic modeling and mitigation approaches. Transportation Research Part B 41 (1), 49-62.

Galor, O., 2007. Discrete dynamical systems. Springer Verlag.

Godfrey, J., 1969. The mechanism of a road network. Traffic Engineering and Control 8 (8), $323-327$.

Greenshields, B. D., 1935. A study of traffic capacity. Highway Research Board Proceedings $14,448-477$.

Haberman, R., 1977. Mathematical models. Prentice Hall, Englewood Cliffs, NJ.

Jin, W.-L., 2012a. A Riemann solver for a system of hyperbolic conservation laws at a general road junction. Arxiv preprint.

URL http://arxiv.org/abs/1204.6727

Jin, W.-L., 2012b. A kinematic wave theory of multi-commodity network traffic flow. Transportation Research Part B 46 (8), 1000-1022.

Jin, W.-L., 2012c. The traffic statics problem in a road network. Transportation Research Part B 46 (10), 1360-1373.

Jin, W.-L., 2013. Stability and bifurcation in network traffic flow: A Poincaré map approach. Transportation Research Part B 57, 191-208.

Jin, W.-L., 2015. On the existence of stationary states in general road networks. Transportation Research Part B 81, 917-929.

Jin, W.-L., Gan, Q.-J., Gayah, V. V., 2013. A kinematic wave approach to traffic statics and dynamics in a double-ring network. Transportation Research Part B 57, 114-131.

LaSalle, J., 1976. The stability of dynamical systems. Society for Industrial Mathematics.

Lebacque, J. P., 1996. The Godunov scheme and what it means for first order traffic flow models. Proceedings of the 13th International Symposium on Transportation and Traffic Theory, 647-678.

Lighthill, M. J., Whitham, G. B., 1955. On kinematic waves: II. A theory of traffic flow on long crowded roads. Proceedings of the Royal Society of London A 229 (1178), 317-345.

Merchant, D., Nemhauser, G., 1978. Optimality conditions for a dynamic traffic assignment model. Transportation Science 12 (3), 200-207. 
Munjal, P. K., Hsu, Y. S., Lawrence, R. L., 1971. Analysis and validation of lane-drop effects of multilane freeways. Transportation Research 5 (4), 257-266.

Newell, G. F., 1993. A simplified theory of kinematic waves in highway traffic I: General theory. II: Queuing at freeway bottlenecks. III: Multi-destination flows. Transportation Research Part B 27 (4), 281-313.

Payne, H., Thompson, W., 1974. Allocation of freeway ramp metering volumes to optimize corridor performance. IEEE Transactions on Automatic Control 19 (3), 177-186.

Potts, R. B., Oliver, R. M., 1972. Flows in transportation networks. Academic Press.

Richards, P. I., 1956. Shock waves on the highway. Operations Research 4 (1), 42-51.

Wattleworth, J., 1967. Peak period analysis and control of a freeway system/with discussion. Highway Research Record 157, 1-21.

Wiggins, S., Heck, A., 2003. Introduction to Applied Nonlinear Dynamical Systems and Chaos. Springer.

Yang, H., Lam, W. H., 1996. Optimal road tolls under conditions of queueing and congestion. Transportation Research Part A 30 (5), 319-332.

Yang, H., Yagar, S., 1995. Traffic assignment and signal control in saturated road networks. Transportation Research Part A 29 (2), 125-139. 